2016/01

A Dynamic Component Model for Forecasting High-Dimensional Realized Covariance Matrices

Luc Bauwens, Manuela Braione, Giuseppe Storti

\title{
50 YEARS OF
}

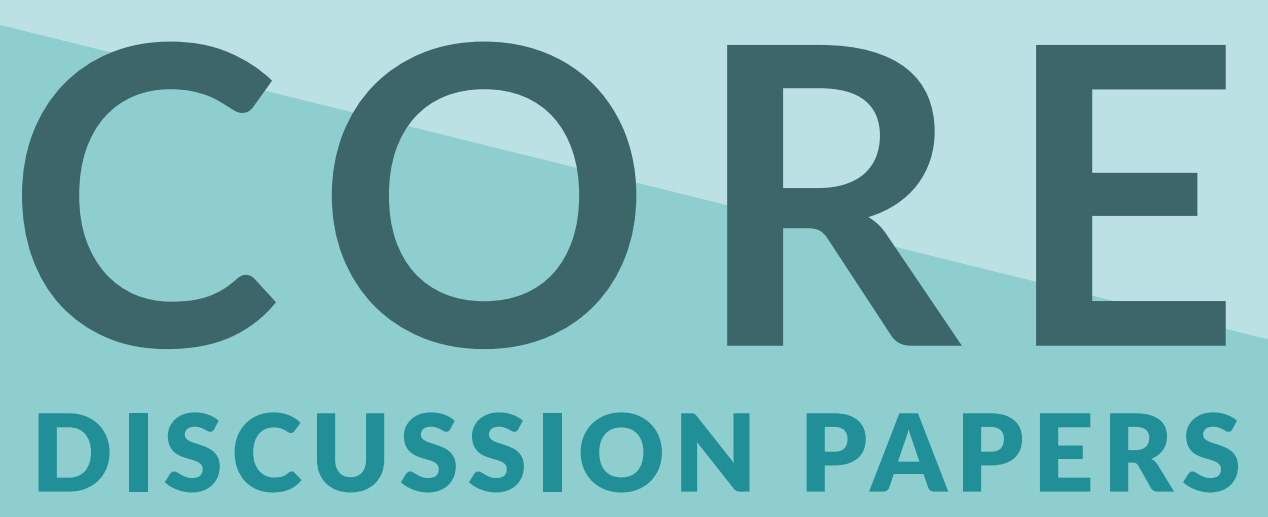




\section{CORE}

Voie du Roman Pays 34, L1.03.01

Tel (32 10) 474304

Fax (32 10) 474301

Email: immaq-library@uclouvain.be

http://www.uclouvain.be/en-44508.html 


\title{
A dynamic component model for forecasting high-dimensional realized covariance matrices
}

\author{
L.Bauwens, M.Braione, G.Storti \\ Université catholique de Louvain, CORE, and SKEMA Business School-Université de Lille, \\ luc.bauwens@uclouvain.be \\ Université catholique de Louvain, CORE, manuela.braione@uclouvain.be \\ University of Salerno, DISES, storti@unisa.it
}

February 1, 2016

\begin{abstract}
The Multiplicative MIDAS Realized DCC (MMReDCC) model of Bauwens et al. [5] decomposes the dynamics of the realized covariance matrix of returns into short-run transitory and long-run secular components where the latter reflects the effect of the continuously changing economic conditions. The model allows to obtain positive-definite forecasts of the realized covariance matrices but, due to the high number of parameters involved, estimation becomes unfeasible for large cross-sectional dimensions. Our contribution in this paper is twofold. First, in order to obtain a computationally feasible estimation procedure, we propose an algorithm that relies on the maximization of an iteratively re-computed moment-based profile likelihood function. We assess the finite sample properties of the proposed algorithm via a simulation study. Second, we propose a bootstrap procedure for generating multi-step ahead forecasts from the MMReDCC model. In an empirical application on realized covariance matrices for fifty equities, we find that the MMReDCC not only statistically outperforms the selected benchmarks in-sample, but also improves the out-of-sample ability to generate accurate multi-step ahead forecasts of the realized covariances.
\end{abstract}

Keywords: Realized covariance, dynamic component models, multi-step forecasting, MIDAS, targeting, model confidence set.

\section{Introduction}

Building models for predicting the volatility of high dimensional portfolios is important in risk management and asset allocation. Previous developments on time-varying covariances in large dimensions include the constant conditional correlation (CCC) model of Bollerslev [8], where the volatilities of each asset are allowed to vary through time but the correlations are time invariant, the RiskMetrics model by [27], and the DECO model by Engle and Kelly [18] who allow correlations to change over time and can be easily applied 
in vast dimensions. Recently, Andersen et al. [2], Barndorff-Nielsen and Shephard [4] and Barndorff-Nielsen et al. [3], among others, opened up a new channel for increasing the precision of covariance matrix estimates and forecasts by exploiting the information of high frequency asset returns. This development has motivated several researchers to investigate models directly fitted to series of realized covariance matrices (see Gouriéroux et al. [22], Jin and Maheu [25] and Chiriac and Voev [11], among others).

Despite the superiority of these models, illustrated for example by Hautsch et al. [24], there still remain technical and practical challenges one needs to deal with when constructing covariance matrix forecasts for high-dimensional systems. First and foremost, the well-known "curse of dimensionality" problem, implying that the number of parameters grows as a power function of the cross-sectional model dimension. In order to save parameters, a simple solution is represented by the so called covariance (or correlation) targeting approach of Engle [15], which consists in pre-estimating the constant intercept matrix in the model specification by linking it to the unconditional covariance matrix of returns. This method can be applied under the stationarity assumption of the model and is one of the most widely employed techniques to simplifying parameter estimation and reducing the computational burden when the numerical maximization of the likelihood function becomes difficult.

Recently, Bauwens et al. [5] investigated a wide class of multivariate models that simultaneously account for short and long-term dynamics in the conditional (co)volatilities and correlations of asset returns, in line with the empirical evidence suggesting that their level is changing over time as a function of the economic conditions (see, among others, Engle et al. [16]). Herein we focus on the Multiplicative MIDAS Realized DCC (MMReDCC) model, whose main ingredients are a multiplicative component structure, a Mixed Data Sampling (MIDAS) filter to modeling the secular dynamics and a DCC-type parameterization for the short term component, directly inspired by the multivariate GARCH literature. ${ }^{1}$ The extensive out-of-sample forecasting comparison performed by Bauwens et al. [5], although not identifying a clear winner, shows that the MMReDCC model gives remarkably good performances in important financial applications such as Value-at-Risk forecasting and portfolio allocation. However, their results are limited to a relatively low dimensional setting (10 assets) and to a short-term forecasting horizon (1 day).

This paper extends the work by Bauwens et al. [5] along these directions: estimation for high-dimensional systems and multi-step forecasting. We contribute to the first line of research by developing of a computationally feasible procedure for the estimation of vast dimensional MMReDCC models. In this respect, it is important to remark that, although the introduction of a dynamic secular component in the structure of the model adds a major element of flexibility and enables to obtain more accurate forecasts than standard models reverting to constant mean levels (see Bauwens et al. [5]), it also dramatically increases the number of parameters to be estimated. Specifically, the long term component incorporates

\footnotetext{
${ }^{1}$ We refer to Engle [14] and Ghysels et al. [20] as leading references for detailed discussions of the DCC model and MIDAS regressions.
} 
a scale intercept matrix with number of parameters equal to $n(n+1) / 2$, where $n$ denotes the number of assets. In a vast dimensional framework, this quickly translates into the impossibility of estimating the model since the intercept matrix cannot be directly targeted.

Therefore, we propose to overcome this estimation issue by proposing an iterative procedure inspired by the covariance targeting of Engle [15]. More precisely, based on a Method of Moments estimator, we profile out the parameters of the intercept matrix and iteratively maximize the likelihood in terms of the other parameters of interest. We refer to this as the Iterative Moment-Based Profiling (IMP) estimator, as opposed to the Quasi Maximum Likelihood (QML) estimator which directly maximizes the likelihood with respect to the full parameter vector.

It is worth noting that the proposed estimation procedure can be considered a switching algorithm in the sense discussed by Boswijk [9] and Cubadda and Scambelloni [13] since the maximization of the overall likelihood is obtained by switching between optimizations over different blocks of parameters. This idea has a long standing tradition in the econometric analysis of time series. A simple, well known example of switching algorithm is given by the Cochrane-Orcutt iterative estimation procedure. Compared to conventional switching algorithms, the procedure that is here implemented incorporates an additional targeting step. In particular, it reduces the dimension of the optimization problem to be solved by concentrating out some of the parameters, the elements of the intercept matrix, by means of an iteratively re-computed moment-based estimator. A comprehensive simulation study is performed to assess the finite-sample properties of the proposed estimator which is found to deliver unbiased estimates and to quickly converge, as no more than three iterations are required in general.

The second relevant contribution of the paper is the development of a resampling based procedure for the generation of multi-step ahead forecasts of the realized covariance matrices. The multiplicative component structure of the MMReDCC model makes the derivation of a closed-form expression for the h-step predictor impossible. Hence, to solve this issue we use a distribution-free procedure based on a residual bootstrap method. The bootstrap has been a standard tool for generating multi-step forecasts from non-linear and non-Gaussian time series models for more than two decades (see e.g. Clements and Smith [12]). Its use has been later extended to univariate volatility modeling (see e.g. Pascual and Ruiz [29]; Shephard and Sheppard [31]). More recently, Fresoli and Ruiz [19] have proposed a simple resampling algorithm that makes use of residual bootstrap to compute multi-step forecasts from DCC models. The bootstrap procedure which is implemented in this paper builds on the work of Fresoli and Ruiz [19] but the algorithm is adapted to the dynamic modeling of realized covariance matrices.

Finally, the results of two different applications to real data are presented and discussed. In the first one, we focus on a low dimensional setting (ten assets), in which both the IMP and one-step QML estimation procedures are feasible, and compare the estimates obtained by means of both algorithms. We find that the IMP-based estimates are sufficiently close to the QML ones, so that using the IMP method in large dimensions is a sensible approach. In the second application the MMReDCC model estimated for fifty assets by the IMP method is used to generate forecasts of the realized covariance matrix, up to twenty days ahead, 
and compared to existing benchmarks not accounting for short and long term (co)volatility dynamics. For the dataset considered, in correspondence of a forecasting horizon equal to one day, the MMReDCC model is outperformed by the benchmarks while it dominates for longer horizons up to ten days.

The remainder of the paper is organized as follows. Section 2 briefly recalls the structure of the MMReDCC model and explains the curse of dimensionality issue. Section 3 introduces the IMP algorithm and Section 4 presents the results of a Monte Carlo experiment aimed at assessing the finite sample statistical properties of the proposed estimation algorithm. The bootstrap procedure for computing multi-step ahead forecasts is explained in Section 5. Section 6 contains the empirical results for the in-sample estimation comparison and the out-of-sample forecasting exercise. Section 7 concludes with some final remarks.

\section{The MMReDCC model}

Let $C_{t}$ be a $n \times n$ positive definite and symmetric (PDS) realized estimator of the latent integrated covariance (IC) matrix of daily returns. In the following, unless otherwise stated, we will refer to $C_{t}$ as the realized covariance $(\mathrm{RC})$, although any other consistent PDS estimator could be used. Conditionally on the set consisting of all relevant information up to and including day $t-1, C_{t}$ is assumed to follow a $n$-dimensional central Wishart distribution:

$$
C_{t} \mid I_{t-1} \sim W_{n}\left(\nu, S_{t} / \nu\right), \quad \forall t=1, \ldots, T,
$$

where $\nu(>n-1)$ is the degrees of freedom parameter and $S_{t}$ is the PDS conditional mean matrix of order $n$. Under the assumption of absence of microstructure noise and other biases (see Barndorff-Nielsen and Shephard [4]), $S_{t}$ represents the conditional covariance matrix of returns, which is our object of interest.

In the MMReDCC model, $S_{t}$ is designed to directly capture the long run movements in the levels around which realized (co)variances (and by extension, correlations) fluctuate from day to day. To this extent, the model features a multiplicative decomposition of the conditional covariance matrix $S_{t}$ into a smoothly varying or secular component $M_{t}=L_{t} L_{t}^{\prime}$ and a short-lived component $S_{t}^{*}$, such that $S_{t}$ can be rewritten as $S_{t}=L_{t} S_{t}^{*} L_{t}^{\prime}$, where the matrix square root $L_{t}$ can be obtained by a Cholesky factorization of $M_{t}$. These components can then be modeled separately.

First, the secular component is specified parametrically and extracted by means of a MIDAS filter assumed to be a weighted sum of $K$ lagged realized covariance matrices over a long horizon, where the number of lags spanned in the MIDAS specification is usually chosen to minimize the trade-off between the highest in-sample likelihood value and the number of observations lost to initialize the filter. It is expressed as

$$
M_{t}=\Lambda+\theta \sum_{k=1}^{K} \phi_{k}(\omega) C_{t-k}
$$


In the right hand side of Eq.(2), the first term $\Lambda$ is a $n \times n$ symmetric and semi-positive definite matrix of constant parameters, $\theta$ is a positive scalar and $\phi_{k}(\cdot)$ is a weight function parametrized according to the restricted Beta polynomial

$$
\phi_{k}(\omega)=\frac{\left(1-\frac{k}{K}\right)^{\omega-1}}{\sum_{j=1}^{K}\left(1-\frac{j}{K}\right)^{\omega-1}},
$$

The scalar parameter $\omega$ dictates the shape of the function and in order to achieve a timedecaying pattern of the weights, it is constrained to be larger than 1. For identification, the constraint $\sum_{k=1}^{K} \phi_{k}(\omega)=1$ is imposed.

Second, the dynamics of the short term component $S_{t}^{*}$ is specified according to a scalar DCC parametrization that enables a separate treatment of conditional volatilities and correlations, thus allowing for a high degree of flexibility. Letting $X$ be any square matrix of arbitrary size $n$, in the remainder the notation $\operatorname{diag}(X)$ is used to denote a $n \times n$ diagonal matrix with non-zero elements equal to the diagonal elements of $X$. Therefore, assuming that $S_{t}^{*}=D_{t}^{*} R_{t}^{*} D_{t}^{*}$, where $D_{t}^{*}=\operatorname{diag}\left\{S_{t}^{*}\right\}^{1 / 2}$, their scalar specifications correspond to the following equations:

$$
\begin{aligned}
S_{i i, t}^{*} & =\left(1-\gamma_{i}-\delta_{i}\right)+\gamma_{i} C_{i i, t-1}^{*}+\delta_{i} S_{i i, t-1}^{*}, \quad \forall i=1, \ldots, n \\
R_{t}^{*} & =(1-\alpha-\beta) I_{n}+\alpha P_{t-1}^{*}+\beta R_{t-1}^{*},
\end{aligned}
$$

where $\gamma_{i}>0, \delta_{i} \geq 0, \gamma_{i}+\delta_{i}<1, \alpha>0, \beta \geq 0, \alpha+\beta<1, C_{t}^{*}=L_{t}^{-1} C_{t}\left(L_{t}^{\prime}\right)^{-1}$ and $P_{t}^{*}=\left(\operatorname{diag}\left\{C_{t}^{*}\right\}\right)^{-1 / 2} C_{t}^{*}\left(\operatorname{diag}\left\{C_{t}^{*}\right\}\right)^{-1 / 2}$. The matrix $C_{t}^{*}$ is the realized covariance matrix purged of its long term component and the matrix $P_{t}^{*}$ is the corresponding short term realized correlation matrix. Mean reversion to unity in Eq.(3) and to an identity matrix in Eq.(4) is needed for identification of the different components. Let $\gamma=\left\{\gamma_{1}, \ldots, \gamma_{n}\right\}$, $\boldsymbol{\delta}=\left\{\delta_{1}, \ldots, \delta_{n}\right\}$ for further use.

The parameters can be estimated by maximizing the following Wishart (quasi) loglikelihood function in one step:

$$
\ell_{T}(\boldsymbol{\psi})=-\frac{1}{2} \sum_{t=1}^{T}\left\{\log \left|S_{t}(\boldsymbol{\psi})\right|+\operatorname{tr}\left[S_{t}(\boldsymbol{\psi})^{-1} C_{t}\right]\right\}
$$

The finite-dimensional parameter vector $\boldsymbol{\psi}=\{\operatorname{vech}(\Lambda), \theta, \omega, \boldsymbol{\gamma}, \boldsymbol{\delta}, \alpha, \beta\}^{2}$, has length $\left\{n_{\Lambda}+\right.$ $2 n+4\}$ where $n_{\Lambda}=n(n+1) / 2=O\left(n^{2}\right)$ denotes the number of unique parameters included in the intercept matrix $\Lambda$ of Eq.(2). It is obvious that, as $n$ increases, the curse of dimensionality problem quickly arises, leading to the number of parameters listed in the first two rows of Table 1 . Observe that estimation becomes already cumbersome after $n=20$ and almost impossible for $n \geq 50$.

\footnotetext{
${ }^{2}$ Note that $\psi$ do not include the degree of freedom parameter $\nu$, as the first order conditions for the estimation of the parameter vector $\psi$ do not depend on $\nu$ by linearity in $\nu$ (see [5]).
} 
Table 1: Number of parameters of MMReDCC models

\begin{tabular}{cccccc}
\hline & $n=5$ & $n=10$ & $n=20$ & $n=50$ & $n=100$ \\
\cline { 2 - 6 }$n_{\Lambda}$ & 15 & 55 & 210 & 1275 & 5050 \\
$\psi$ & 29 & 79 & 254 & 1379 & 5254 \\
$\tilde{\psi}$ & 14 & 24 & 44 & 104 & 204 \\
\hline
\end{tabular}

Note: Entries report the number of parameters as a function of the dimension $n ; n_{\Lambda}$ denotes the number of unique parameters contained in the $\Lambda$ matrix, $\psi$ denotes the full vector of model parameters and $\tilde{\psi}$ the vector of parameters excluding $n_{\Lambda}$.

On the other hand, the last row of Table 1 shows that an intuitive way to keep the model tractable is to avoid estimating the parameters of the matrix $\Lambda$. This would be sufficient to reduce the order to $2 n+4=O(n)$, thus making the model estimable also for large $n$.

In the following section we put forward a feasible estimation procedure that aims at overcoming the direct estimation of the long term component intercept matrix, thus crucially mitigating the computational complexity of the model.

\section{An Iterative Moment based Profiling (IMP) algorithm}

In this section we discuss an iterative procedure for fitting the MMReDCC model to large dimensional datasets. The basic idea underlying the proposed algorithm is to eliminate from the likelihood maximization the parameters of the intercept matrix $\Lambda$ using a technique that builds upon the covariance targeting discussed in Pedersen and Rahbek [30] for BEKK and Engle et al. [17] for DCC models. First of all, notice that from Eq.(2) and the following relation

$$
\Lambda=E\left(M_{t}\right)-\theta \sum_{k=1}^{K} \phi_{k}(\omega) E\left(C_{t-k}\right),
$$

a moment based estimator of the $\Lambda$ intercept matrix is

$$
\hat{\Lambda}=\frac{1}{T} \sum_{t=1}^{T}\left[M_{t}-\theta \sum_{k=1}^{K} \phi_{k}(\omega) C_{t-k}\right] \text {. }
$$

Obviously, given the latent nature of $M_{t}$, the estimator in Eq.(6) cannot be computed in practice and hence the covariance targeting approach cannot be applied in the usual way. It is worth noting that, if $L_{t}$ and $S_{t}^{*}$ were assumed to be independent, given $E\left(S_{t}^{*}\right)=I_{n}$, it would hold that $E\left(C_{t}\right)=E\left(M_{t}\right)$, implying that an asymptotically equivalent version of Eq.(6) could be explicitly computed replacing $M_{t}$ by $C_{t}$. However, this is not the approach we pursue, since the assumption of independence of the short and long term sources is 
difficult to justify and would result in a rather counterintuitive and arbitrary constraint. Hence, we adopt a different method.

By noting from Eq.(6) that no estimate of $\Lambda$ makes sense regardless of the value of $(\theta, \omega)$, we make this dependence explicit and obtain an estimate of $\Lambda$ as a function of $(\theta, \omega)$, i.e. $\hat{\Lambda}(\theta, \omega)$. In this way, a different estimate of $\Lambda$ is required for each different value of the other two parameters. Therefore, by substituting $\hat{\Lambda}(\theta, \omega)$ for $\Lambda$ in the Wishart QML function stated in Eq.(5), the following moment based QML approximation is obtained:

$$
\tilde{\ell}_{T}(\tilde{\boldsymbol{\psi}})=-\frac{1}{2} \sum_{t=1}^{T}\left\{\log \left|\tilde{L}_{t}(\theta, \omega) S_{t}^{*}(\tilde{\boldsymbol{\psi}}) \tilde{L}_{t}^{\prime}(\theta, \omega)\right|+\operatorname{tr}\left\{\left[\tilde{L}_{t}(\theta, \omega) S_{t}^{*}(\tilde{\boldsymbol{\psi}}) \tilde{L}_{t}^{\prime}(\theta, \omega)\right]^{-1} C_{t}\right\}\right\}
$$

with $\tilde{\boldsymbol{\psi}}=\left(\omega, \theta, \boldsymbol{\psi}_{S^{*}}\right)^{\prime}, \boldsymbol{\psi}_{S^{*}}^{\prime}=(\boldsymbol{\gamma}, \boldsymbol{\delta}, \alpha, \beta)$ and

$$
\tilde{M}_{t}(\theta, \omega)=\tilde{L}_{t}(\theta, \omega) \tilde{L}_{t}^{\prime}(\theta, \omega)=\hat{\Lambda}(\theta, \omega)+\theta \sum_{k=1}^{K} \phi_{k}(\omega) C_{t-k}
$$

The method we propose consists of estimating the parameters in $\tilde{\boldsymbol{\psi}}$ by a block-wise maximization of the moment-based QML function given in Eq.(7). First, conditional on some reasonable initial guess of $(\theta, \omega), \tilde{\ell}_{T}(\tilde{\boldsymbol{\psi}})$ is maximized with respect to the short term parameters $\psi_{S^{*}}$ and then, conditional on $\hat{\boldsymbol{\psi}}_{S^{*}}$, the same function is maximized with respect to $(\theta, \omega)$. The procedure is iterated for $j=0, \ldots, J$ until some pre-specified convergence criterion is met.

To initialize the algorithm at $j=0$, one can reasonably use as starting values the parameter estimates obtained by fitting the model to low dimensional subsets of data; also, an initial guess for the long term component $M_{t, 0}$ could be either provided in a naive way, i.e. using the series of observed realized covariance matrices directly, or in a more sophisticated manner, by fitting to the data a nonparametric kernel smoother with an optimized bandwidth parameter. Note that in order to guarantee the positive definiteness of $\tilde{M}_{t}(\theta, \omega)$ in Eq. (8), it suffices to initialize $M_{t, 0}$ from a PDS matrix and to impose $\theta>0$. Given that the observed series of $C_{t}$, for every $t$, is PDS by definition, $\hat{\Lambda}(\theta, \omega)$ is assured to be at least semi-positive definite at each iteration $j>0$.

Once $\Lambda_{j}\left(\theta_{j}, \omega_{j}\right)$ has been computed at the initial iteration $j=0$, for every $j>0$ the steps conducted in the algorithm are as follows:

Step 1 Plug $\Lambda_{j-1}\left(\theta_{j-1}, \omega_{j-1}\right)$ into Eq. (2), then get $\tilde{M}_{t, j}$ and $\tilde{L}_{t, j}=\operatorname{chol}\left(\tilde{M}_{t, j}\right)$ for all $t$;

Step 2 For each asset $i=1, \ldots n$, obtain the short term $\operatorname{GARCH}(1,1)$ parameters of Eq.(3) as follows

$$
\left\{\gamma_{i, j}, \delta_{i, j}\right\}=\underset{\left\{\gamma_{i}, \delta_{i}\right\}}{\arg \max } \quad \tilde{\ell}_{T}\left(\theta_{j-1}, \omega_{j-1}, \alpha_{j-1}, \beta_{j-1}\right) ;
$$

Step 3 Conditional on the estimated vectors $\gamma_{j}=\left(\gamma_{1, j}, \ldots, \gamma_{n, j}\right)^{\prime}$ and $\boldsymbol{\delta}_{j}=\left(\delta_{1, j}, \ldots, \delta_{n, j}\right)^{\prime}$, maximize the same log-likelihood function with respect to the short term DCC correlation parameters:

$$
\left\{\alpha_{j}, \beta_{j}\right\}=\underset{\{\alpha, \beta\}}{\arg \max } \quad \tilde{\ell}_{T}\left(\theta_{j-1}, \omega_{j-1}, \boldsymbol{\gamma}_{j}, \boldsymbol{\delta}_{j}\right) ;
$$


Step 4 Finally, conditional on the vector of short term parameter estimates $\boldsymbol{\phi}_{S^{*}}=\left\{\boldsymbol{\gamma}_{j}, \boldsymbol{\delta}_{j}, \alpha_{j}\right.$, $\left.\beta_{j}\right\}$, maximize $\tilde{\ell}_{T}$ with respect to $\left\{\theta_{j}, \omega_{j}\right\}$; these estimates are used to compute an updated version of $\Lambda_{j}\left(\theta_{j}, \omega_{j}\right)$;

Step 5 Check for convergence otherwise update all parameter estimates and go back to Step 1.

It is worth to stress that although $\tilde{\ell}_{T}(\tilde{\boldsymbol{\psi}})$ looks like a profile likelihood, it is not since $\hat{\Lambda}(\theta, \omega)$ is not a QML estimator but a feasible moment estimator. This motivates our choice to refer to Steps $1-5$ as the Iterative Moment based Profiling algorithm, or IMP for short. This implies that $\tilde{\boldsymbol{\psi}}$ is typically less efficient than the standard QML estimator that maximizes Eq.(5) in one step. We come back to this issue in Section 6.1.

\section{Simulation study}

A Monte Carlo study is conducted to analyse the finite sample properties of the IMP estimator. We assume the MMReDCC to be the DGP and we generate 500 processes of length $T=1000$ and 2000 for $n=10,20,40$ and 50, with true parameter values inspired by the estimates given in Bauwens et al. [5], as summarized in Table 2.

It is important to stress that, in order to initialize the algorithm, parameter values have to be carefully chosen. This is a standard requirement in every optimization based procedure where the initial amount of information on the model parameters can be limited or even null. In our situation we are mainly concerned with the impact that different choices of $M_{t, 0}$, more than the remaining set of parameters, may have on the convergence of the IMP algorithm. We evaluate this by performing a robustness check based on the two possible initializations of $M_{t, 0}$ mentioned in Section 3 .

In the first set of repetitions $M_{t, 0}$ is computed by fitting to the series of simulated realized covariance matrices a Nadaraya-Watson kernel estimator with a single bandwidth parameter for the whole covariance matrix. As in Bauwens et al. [5] and Bauwens et al. [6], the optimal bandwidth is selected by a least squares cross-validation criterion, where the six-month rolling covariance is used as the reference for the computation of least squares. In the second (equivalent) simulation study, $M_{t, 0}$ is obtained by substituting in Eq. (6) the observed $C_{t}$ for the latent matrix $M_{t}$ at each $t$. In both cases, the initial scalar model parameters are set equal to the values listed in Panel B of Table 2.

The estimation bias is evaluated by the relative bias (RB), computed as $\frac{1}{500} \sum_{i=1}^{500} \frac{\hat{\psi}_{i}-\psi}{\psi}$, along with the interquartile range (IQR), mean, minimum and maximum of the obtained parameter estimates. To save space, we report averaged bias results for the parameters of the MIDAS intercept matrix in a separate table.

Table 3 reports results from the first simulation exercise. The emerging picture looks encouraging. As expected, the relative biases decrease as $n$ or $T$ increases. The biases for the parameters of the short term volatility and correlation components are very small, being smaller than five per cent in most of the cases, with one exception recorded for $\bar{\gamma}$ at $T=1000$ for $n=10$. As for the scalar parameters in the MIDAS specification, the bias 
Table 2: Simulation setting

\begin{tabular}{|c|c|c|c|c|}
\hline \multicolumn{5}{|c|}{ Panel A: Parameters } \\
\hline \multicolumn{5}{|c|}{ Long term component } \\
\hline \multicolumn{3}{|l|}{$\theta$} & \multicolumn{2}{|l|}{0.5} \\
\hline \multicolumn{3}{|l|}{$K$} & \multicolumn{2}{|l|}{264} \\
\hline \multicolumn{3}{|l|}{$\omega$} & \multicolumn{2}{|l|}{15} \\
\hline \multicolumn{3}{|l|}{$\Lambda$} & \multicolumn{2}{|c|}{$\Lambda_{i, i}=0.02, \Lambda_{i, j}=0.002$ for $i \neq j$} \\
\hline \multicolumn{5}{|c|}{ Short term components } \\
\hline \multicolumn{3}{|c|}{$\gamma_{i}$} & \multicolumn{2}{|c|}{$\sim U\left(\gamma_{0}-0.02, \gamma_{0}+0.02\right), \gamma_{0}=0.2$} \\
\hline \multicolumn{3}{|l|}{$\delta_{i}$} & \multicolumn{2}{|c|}{$\sim U\left(2 \delta_{0}+\gamma_{i}-1+0.01,1-\gamma_{i}-0.01\right), \delta_{0}=0.7$} \\
\hline \multicolumn{3}{|l|}{$\alpha$} & \multicolumn{2}{|c|}{0.2} \\
\hline \multicolumn{3}{|l|}{$\beta$} & \multicolumn{2}{|l|}{0.7} \\
\hline \multicolumn{5}{|c|}{ General } \\
\hline \multicolumn{3}{|l|}{$\nu$} & \multicolumn{2}{|l|}{$2 n$} \\
\hline \multicolumn{3}{|l|}{$\mathrm{T}$} & \multicolumn{2}{|l|}{1000,2000} \\
\hline \multicolumn{3}{|c|}{ initial discarded observations } & \multicolumn{2}{|l|}{1000} \\
\hline \multicolumn{3}{|c|}{ convergence tolerance } & \multicolumn{2}{|l|}{0.0001} \\
\hline \multicolumn{5}{|c|}{ Panel B: Initial values } \\
\hline$\theta_{0}$ & $\omega_{0}$ & $\gamma_{i, 0}$ & $\delta_{i, 0}$ & $\beta_{0}$ \\
\hline 0.8 & 10 & 0.05 & 0.90 & 0.9 \\
\hline
\end{tabular}

Note: In Panel A, for every $i=1, \ldots, n$ it holds $\left\{\gamma_{i}+\delta_{i}\right\}<1$. Entries of Panel B are scalar parameters chosen to initialize the algorithm in both sets of simulation exercises.

for $\theta$ is negative in seven out of eight cases (the exception occurs for $n=10$ at $T=2000$ ) and ranging from the maximum of $5.8 \%$ (in absolute value) for $n=10$ and $T=1000$ to the lowest value of $0.1 \%$ for $n=50$ and $T=2000$. The bias on the $\omega$ parameter, also generally negative, tends to decrease with $n$ but is usually of higher order (from 1.1 to $12 \%$ in absolute value). A similar behavior is observed for the IQR measure, which decreases across $n$ and $T$ but remains on higher values for the parameter $\omega$. However, this does not represent a major concern as the Beta weight function is not very sensitive to small variations of this parameter and therefore we do not expect the likelihood function to be either.

Table 4 gives an idea of the robustness of the results to the other initialization of the long term component. Entries can be directly compared to those in Table 3. As hoped for, the initial choice has a minor impact on the overall accuracy of the estimator, as the parameter biases are in the same range of magnitude and the comments made earlier are still valid under this alternative scenario. Figure C.2 contains plots of the Monte Carlo standard deviations of the estimated $\theta, \omega, \alpha$ and $\beta$ parameters against the cross-section size. 
Table 3: Simulation exercise I: summary statistics

\begin{tabular}{|c|c|c|c|c|c|c|}
\hline \multicolumn{7}{|c|}{$T=1000$} \\
\hline & $\bar{\gamma}$ & $\bar{\delta}$ & $\alpha$ & $\beta$ & $\theta$ & $\omega$ \\
\hline & 0.197 & 0.705 & 0.2 & 0.7 & 0.5 & 15 \\
\hline \multicolumn{7}{|c|}{$n=10$} \\
\hline $\mathrm{RB}$ & 0.098 & -0.036 & 0.020 & 0.003 & -0.058 & -0.120 \\
\hline IQR & 0.048 & 0.093 & 0.006 & 0.010 & 0.044 & 1.641 \\
\hline Mean & 0.202 & 0.699 & 0.204 & 0.702 & 0.475 & 14.820 \\
\hline Min & 0.176 & 0.660 & 0.191 & 0.679 & 0.393 & 7.460 \\
\hline Max & 0.214 & 0.735 & 0.220 & 0.728 & 0.709 & 18.943 \\
\hline \multicolumn{7}{|c|}{$n=20$} \\
\hline $\mathrm{RB}$ & 0.049 & -0.009 & 0.019 & 0.001 & -0.056 & -0.110 \\
\hline IQR & 0.046 & 0.083 & 0.003 & 0.006 & 0.019 & 0.713 \\
\hline Mean & 0.202 & 0.701 & 0.204 & 0.701 & 0.472 & 14.782 \\
\hline Min & 0.171 & 0.633 & 0.197 & 0.687 & 0.430 & 12.802 \\
\hline Max & 0.219 & 0.744 & 0.211 & 0.711 & 0.532 & 16.759 \\
\hline \multicolumn{7}{|c|}{$\mathrm{n}=40$} \\
\hline $\mathrm{RB}$ & 0.028 & 0.023 & 0.015 & 0.002 & -0.049 & -0.080 \\
\hline IQR & 0.042 & 0.077 & 0.002 & 0.002 & 0.011 & 0.372 \\
\hline Mean & 0.208 & 0.715 & 0.203 & 0.701 & 0.476 & 14.810 \\
\hline Min & 0.190 & 0.656 & 0.060 & 0.695 & 0.446 & 3.735 \\
\hline Max & 0.217 & 0.762 & 0.222 & 0.799 & 0.705 & 16.500 \\
\hline \multicolumn{7}{|c|}{$\mathrm{n}=50$} \\
\hline $\mathrm{RB}$ & 0.027 & 0.011 & 0.016 & 0.001 & -0.045 & 0.012 \\
\hline IQR & 0.042 & 0.076 & 0.001 & 0.002 & 0.008 & 0.293 \\
\hline Mean & 0.208 & 0.716 & 0.203 & 0.701 & 0.473 & 15.182 \\
\hline Min & 0.162 & 0.644 & 0.200 & 0.697 & 0.455 & 13.150 \\
\hline Max & 0.220 & 0.814 & 0.207 & 0.705 & 0.525 & 15.830 \\
\hline
\end{tabular}

\begin{tabular}{|c|c|c|c|c|c|c|}
\hline & \multicolumn{6}{|c|}{$T=2000$} \\
\hline & $\bar{\gamma}$ & $\bar{\delta}$ & $\alpha$ & $\beta$ & $\theta$ & $\omega_{2}$ \\
\hline & 0.197 & 0.705 & 0.2 & 0.7 & 0.5 & 15 \\
\hline \multicolumn{7}{|c|}{$\mathrm{n}=10$} \\
\hline RB & -0.039 & -0.002 & 0.033 & 0.003 & 0.053 & -0.095 \\
\hline IQR & 0.032 & 0.068 & 0.006 & 0.008 & 0.037 & 1.819 \\
\hline Mean & 0.2 & 0.713 & 0.207 & 0.702 & 0.526 & 13.58 \\
\hline Min & 0.153 & 0.669 & 0.183 & 0.523 & 0.072 & 1.949 \\
\hline Max & 0.22 & 0.803 & 0.37 & 0.817 & 1.000 & 17.74 \\
\hline \multicolumn{7}{|c|}{$\mathrm{n}=\mathbf{2 0}$} \\
\hline RB & 0.036 & 0.011 & 0.024 & 0.002 & -0.014 & -0.080 \\
\hline IQR & 0.031 & 0.079 & 0.002 & 0.004 & 0.015 & 0.622 \\
\hline Mean & 0.209 & 0.708 & 0.205 & 0.702 & 0.496 & 13.801 \\
\hline Min & 0.197 & 0.678 & 0.200 & 0.694 & 0.001 & 2.440 \\
\hline Max & 0.221 & 0.739 & 0.220 & 0.748 & 0.598 & 15.270 \\
\hline \multicolumn{7}{|c|}{$\mathrm{n}=40$} \\
\hline RB & 0.033 & 0.029 & 0.022 & 0.002 & -0.014 & -0.072 \\
\hline IQR & 0.030 & 0.064 & 0.001 & 0.002 & 0.007 & 0.263 \\
\hline Mean & 0.209 & 0.719 & 0.204 & 0.702 & 0.493 & 13.925 \\
\hline Min & 0.181 & 0.671 & 0.182 & 0.674 & 0.172 & 1.000 \\
\hline Max & 0.221 & 0.761 & 0.223 & 0.744 & 0.837 & 14.760 \\
\hline \multicolumn{7}{|c|}{$\mathrm{n}=50$} \\
\hline RB & 0.029 & 0.027 & 0.017 & 0.001 & -0.011 & -0.037 \\
\hline IQR & 0.030 & 0.056 & 0.001 & 0.002 & 0.007 & 0.220 \\
\hline Mean & 0.208 & 0.720 & 0.203 & 0.701 & 0.494 & 14.442 \\
\hline Min & 0.191 & 0.657 & 0.199 & 0.695 & 0.474 & 12.089 \\
\hline Max & 0.222 & 0.763 & 0.207 & 0.707 & 0.529 & 16.238 \\
\hline
\end{tabular}

Note: Summary statistics of the first set of simulations where $M_{t, 0}$ is initialized using a nonparametric kernel estimator, see Section 3. To save on space, $\bar{\gamma}$ and $\bar{\delta}$ are reported as averaged values across series and replications. RB denotes the Relative Bias computed over 500 replications. True parameter values used to simulate the process at the top of the table.

In all cases, standard deviations tend to decline as the cross-section dimension grows, with a faster decline when $T=2000$. The two approaches produce similar parameter standard deviations, with slightly bigger values recorded for $\theta$ and $\omega$ under the second simulation experiment in correspondence with the higher cross-section sizes.

If we move to analyzing the bias results for the scale MIDAS intercept matrix, Table 5 shows that under both sets of simulation exercises the estimator $\hat{\Lambda}(\theta, \omega)$ well approximates the true $\Lambda$ matrix at all cross-section dimensions, with the parameter bias (averaged across diagonal and off-diagonal elements) clearly improving with increasing $n$ and $T$. Again, the direct comparison of Panels $\mathrm{A}$ and $\mathrm{B}$ confirms that the algorithm initialized from the series 
Table 4: Simulation exercise II: summary statistics

\begin{tabular}{|c|c|c|c|c|c|c|c|c|c|c|c|c|c|}
\hline \multicolumn{7}{|c|}{$T=1000$} & & \multicolumn{6}{|c|}{$\mathrm{T}=2000$} \\
\hline & $\bar{\gamma}$ & $\bar{\delta}$ & $\alpha$ & $\beta$ & $\theta$ & $\omega$ & & $\bar{\gamma}$ & $\bar{\delta}$ & $\alpha$ & $\beta$ & $\theta$ & $\omega_{2}$ \\
\hline & 0.197 & 0.705 & 0.2 & 0.7 & 0.5 & 15 & & 0.197 & 0.705 & 0.2 & 0.7 & 0.5 & 15 \\
\hline \multicolumn{7}{|c|}{$n=10$} & \multicolumn{6}{|c|}{$\mathrm{n}=10$} & \\
\hline $\mathrm{RB}$ & -0.077 & 0.013 & 0.019 & 0.002 & -0.032 & -0.007 & $\mathrm{RB}$ & 0.043 & 0.006 & 0.025 & 0.002 & -0.012 & -0.069 \\
\hline IQR & 0.070 & 0.113 & 0.007 & 0.010 & 0.043 & 1.664 & IQR & 0.028 & 0.046 & 0.004 & 0.007 & 0.024 & 0.870 \\
\hline Mean & 0.191 & 0.708 & 0.204 & 0.701 & 0.484 & 14.892 & Mean & 0.211 & 0.707 & 0.205 & 0.701 & 0.494 & 13.959 \\
\hline Min & 0.107 & 0.588 & 0.191 & 0.678 & 0.393 & 6.678 & Min & 0.189 & 0.654 & 0.196 & 0.686 & 0.435 & 9.614 \\
\hline Max & 0.231 & 0.833 & 0.218 & 0.723 & 0.927 & 19.237 & Max & 0.232 & 0.748 & 0.213 & 0.715 & 0.623 & 15.535 \\
\hline \multicolumn{7}{|c|}{$\mathrm{n}=20$} & \multicolumn{6}{|c|}{$\mathrm{n}=20$} & \\
\hline $\mathrm{RB}$ & 0.048 & -0.002 & 0.018 & 0.002 & -0.045 & -0.008 & $\mathrm{RB}$ & 0.006 & 0.039 & 0.023 & 0.002 & -0.011 & -0.064 \\
\hline IQR & 0.042 & 0.085 & 0.003 & 0.005 & 0.021 & 0.719 & IQR & 0.030 & 0.060 & 0.002 & 0.004 & 0.013 & 0.510 \\
\hline Mean & 0.207 & 0.702 & 0.204 & 0.701 & 0.477 & 14.887 & Mean & 0.206 & 0.721 & 0.205 & 0.701 & 0.494 & 14.047 \\
\hline Min & 0.196 & 0.649 & 0.197 & 0.689 & 0.429 & 6.751 & Min & 0.164 & 0.680 & 0.199 & 0.692 & 0.467 & 11.108 \\
\hline Max & 0.220 & 0.740 & 0.214 & 0.713 & 0.887 & 16.573 & Max & 0.225 & 0.772 & 0.209 & 0.710 & 0.555 & 15.241 \\
\hline \multicolumn{7}{|c|}{$\mathrm{n}=40$} & \multicolumn{6}{|c|}{$\mathrm{n}=40$} & \\
\hline RB & 0.046 & 0.017 & 0.017 & 0.003 & -0.045 & 0.005 & RB & 0.050 & 0.016 & 0.021 & 0.002 & -0.012 & -0.057 \\
\hline IQR & 0.042 & 0.080 & 0.002 & 0.003 & 0.010 & 0.421 & IQR & 0.029 & 0.053 & 0.001 & 0.002 & 0.007 & 0.245 \\
\hline Mean & 0.209 & 0.713 & 0.203 & 0.702 & 0.477 & 15.078 & Mean & 0.210 & 0.718 & 0.204 & 0.701 & 0.494 & 14.148 \\
\hline Min & 0.197 & 0.682 & 0.193 & 0.696 & 0.116 & 6.895 & Min & 0.194 & 0.658 & 0.202 & 0.697 & 0.479 & 8.433 \\
\hline Max & 0.219 & 0.756 & 0.216 & 0.807 & 0.955 & 49.985 & Max & 0.223 & 0.768 & 0.212 & 0.708 & 0.726 & 14.638 \\
\hline \multicolumn{7}{|c|}{$\mathrm{n}=50$} & \multicolumn{6}{|c|}{$\mathrm{n}=50$} & \\
\hline $\mathrm{RB}$ & 0.029 & 0.028 & 0.016 & 0.002 & -0.053 & 0.015 & $\mathrm{RB}$ & 0.028 & 0.018 & 0.020 & 0.002 & -0.017 & -0.048 \\
\hline IQR & 0.041 & 0.071 & 0.001 & 0.002 & 0.009 & 0.342 & IQR & 0.030 & 0.061 & 0.001 & 0.001 & 0.005 & 0.195 \\
\hline Mean & 0.208 & 0.715 & 0.203 & 0.701 & 0.473 & 15.225 & Mean & 0.208 & 0.721 & 0.204 & 0.701 & 0.492 & 14.278 \\
\hline Min & 0.190 & 0.632 & 0.200 & 0.698 & 0.457 & 14.190 & Min & 0.184 & 0.674 & 0.202 & 0.698 & 0.437 & 11.178 \\
\hline Max & 0.220 & 0.766 & 0.206 & 0.705 & 0.494 & 15.844 & Max & 0.216 & 0.766 & 0.209 & 0.713 & 0.508 & 14.742 \\
\hline
\end{tabular}

Note: Summary statistics of the second set of simulations where $M_{t, 0}$ is initialized from the series of realized covariance matrices, see Section 3. To save on space, $\bar{\gamma}$ and $\bar{\delta}$ are reported as averaged values across series and replications. RB denotes the Relative Bias computed over 500 replications. True parameter values used to simulate the process at the top of the table.

of realized covariance matrices overall performs no worse than the one initialized from a nonparametric smoother.

To summarize, the simulation study carried out in this section suggests that the proposed algorithm works quite accurately in finite samples and converges irrespective of the initialization choice made. Overall, the moment-based estimator used for iteratively targeting the constant intercept matrix in the secular component does not create a severe bias problem in the estimation of the other parameters, thus representing a feasible solution to alleviate the curse of dimensionality issue that would otherwise prevent the use of the MMReDCC model in high dimensional applications. Both initialization methods for $M_{t, 0}$ 
Table 5: Bias results for the scale MIDAS intercept matrix.

\begin{tabular}{|c|c|c|c|}
\hline \multicolumn{2}{|c|}{$\mathrm{T}=1000$} & \multicolumn{2}{|c|}{$\mathrm{T}=\mathbf{2 0 0 0}$} \\
\hline \multicolumn{2}{|c|}{$n=10$} & \multicolumn{2}{|c|}{$\mathrm{n}=10$} \\
\hline $\mathbf{R B}_{\{\mathbf{i}, \mathbf{i}\}}$ & 0.080 & $\mathrm{RB}_{\{\mathrm{i}, \mathrm{i}\}}$ & 0.033 \\
\hline $\mathbf{R B}_{\{\mathbf{i}, \mathrm{j}\}}$ & 0.079 & $\mathbf{R B}_{\{\mathbf{i}, \mathbf{j}\}}$ & 0.000 \\
\hline \multicolumn{2}{|c|}{$n=20$} & \multicolumn{2}{|c|}{$\mathrm{n}=\mathbf{2 0}$} \\
\hline $\mathrm{RB}_{\{, \mathbf{i}, \mathbf{i}}$ & 0.073 & $\mathrm{RB}_{\{\mathrm{i}, \mathrm{i}\}}$ & 0.068 \\
\hline $\mathbf{R B}_{\{\mathbf{i}, \mathrm{j}\}}$ & 0.062 & $\mathrm{RB}_{\{\mathrm{i}, \mathrm{j}\}}$ & 0.048 \\
\hline \multicolumn{2}{|c|}{$\mathrm{n}=40$} & \multicolumn{2}{|c|}{$\mathrm{n}=40$} \\
\hline $\mathbf{R B}_{\{\mathbf{i}, \mathbf{i}\}}$ & 0.073 & $\mathrm{RB}_{\{\mathrm{i}, \mathrm{i}\}}$ & 0.062 \\
\hline $\mathrm{RB}_{\{\mathbf{i}, \mathrm{j}\}}$ & 0.061 & $\mathbf{R B}_{\{\mathrm{i}, \mathrm{j}\}}$ & 0.046 \\
\hline \multicolumn{2}{|c|}{$\mathrm{n}=50$} & \multicolumn{2}{|c|}{$\mathrm{n}=50$} \\
\hline $\mathrm{RB}_{\{\mathbf{i}, \mathbf{i}\}}$ & 0.072 & $\mathbf{R B}_{\{\mathrm{i}, \mathrm{i}\}}$ & 0.004 \\
\hline $\mathrm{RB}_{\{\mathrm{i}, \mathrm{j}\}}$ & 0.057 & $\mathbf{R B}_{\{\mathbf{i}, \mathbf{j}\}}$ & 0.037 \\
\hline
\end{tabular}

\begin{tabular}{lccc}
\multicolumn{3}{c}{ Panel B: Simulation exercise II } \\
\hline & $\mathbf{T}=\mathbf{1 0 0 0}$ & \multicolumn{2}{c}{$\mathbf{T}=\mathbf{2 0 0 0}$} \\
\hline & $\mathbf{n}=\mathbf{1 0}$ & \multicolumn{2}{c}{$\mathbf{n}=\mathbf{1 0}$} \\
\hline $\mathbf{R B}_{\{\mathbf{i}, \mathbf{i}\}}$ & 0.075 & $\mathbf{R B}_{\{\mathbf{i}, \mathbf{i}\}}$ & 0.060 \\
$\mathbf{R B}_{\{\mathbf{i}, \mathbf{j}\}}$ & 0.066 & $\mathbf{R B}_{\{\mathbf{i}, \mathbf{j}\}}$ & 0.040 \\
\hline & $\mathbf{n}=\mathbf{2 0}$ & \multicolumn{2}{c}{$\mathbf{n}=\mathbf{2 0}$} \\
\hline $\mathbf{R B}_{\{\mathbf{i}, \mathbf{i}\}}$ & 0.072 & $\mathbf{R B}_{\{\mathbf{i}, \mathbf{i}\}}$ & 0.058 \\
$\mathbf{R B}_{\{\mathbf{i}, \mathbf{j}\}}$ & 0.060 & $\mathbf{R B}_{\{\mathbf{i}, \mathbf{j}\}}$ & 0.044 \\
\hline & $\mathbf{n}=\mathbf{4 0}$ & \multicolumn{2}{c}{$\mathbf{n}=\mathbf{4 0}$} \\
\hline $\mathbf{R B}_{\{\mathbf{i}, \mathbf{i}\}}$ & 0.073 & $\mathbf{R B}_{\{\mathbf{i}, \mathbf{i}\}}$ & 0.058 \\
$\mathbf{R B}_{\{\mathbf{i}, \mathbf{j}\}}$ & 0.159 & $\mathbf{R B}_{\{\mathbf{i}, \mathbf{j}\}}$ & 0.043 \\
\hline & $\mathbf{n}=\mathbf{5 0}$ & \multicolumn{2}{c}{$\mathbf{n}=\mathbf{5 0}$} \\
\hline $\mathbf{R B}_{\{\mathbf{i}, \mathbf{i}\}}$ & 0.072 & $\mathbf{R B}_{\{\mathbf{i}, \mathbf{i}\}}$ & 0.058 \\
$\mathbf{R B}_{\{\mathbf{i}, \mathbf{j}\}}$ & 0.058 & $\mathbf{R B}_{\{\mathbf{i}, \mathbf{j}\}}$ & 0.043 \\
\hline
\end{tabular}

Note: $R B_{\{i, i\}}$ denotes averaged values over diagonal terms, while $R B_{\{i, j\}}$ denotes averages over off diagonal terms. Panel (a) reports summary statistics of the first simulation exercise where $M_{t, 0}$ is initialized from a nonparametric smoother while Panel (b) reports results from the second simulation exercise where the series of observed realized covariance matrices are used.

can be used in practice. In the empirical section, we have opted for the nonparametric smoother.

\section{Multi-step Forecasting}

Models featuring short and long-run dynamics are particularly attractive for computing multi-step-ahead predictions, as their component dynamic structure is possibly expected to be beneficial for longer-term forecasts. The complex nonlinear structure of the MMReDCC model makes the analytical derivation of closed-form solutions impossible. In order to overcome this problem, we propose to compute multi-step predictions by means of a procedure based on bootstrap resampling.

At the outset, notice that Eq.(1) implies that $E\left(C_{t} \mid \Im_{t-1}\right)=S_{t}$, so that $C_{t}$ can be represented as

$$
C_{t}=S_{t}^{1 / 2} U_{t}\left(S_{t}^{1 / 2}\right)^{\prime}
$$

where $U_{t}$ is an element of a sequence of iid random matrices such that $E\left(U_{t}\right)=I_{n}$, and $S_{t}^{1 / 2}$ is any PDS matrix such that $S_{t}^{1 / 2}\left(S_{t}^{1 / 2}\right)^{\prime}=S_{t}$. The Wishart assumption of Eq.(1) is recovered if $U_{t} \sim W_{n}\left(\nu, I_{n} / \nu\right)$, but this assumption is not needed to justify the bootstrap procedure that we use for generating multi-step-ahead forecasts of the realized covariance matrix $C_{t}$. The procedure is described in the following six steps. 
Step 1 Estimate the model on $\left\{C_{t}, t=1, \ldots, T\right\}$ and obtain the estimated conditional covariances $\hat{S}_{t}$.

Step 2 Compute the estimated residuals

$$
\hat{U}_{t}=\hat{S}_{t}^{-1 / 2} C_{t}\left(\hat{S}_{t}^{-1 / 2}\right)^{\prime}, \quad t=1, \ldots, T
$$

and rescale them to enforce their sample mean to be equal to $I_{n}$, namely:

$$
\tilde{U}_{t}=\left(\hat{E}_{u}^{-1 / 2}\right) \hat{U}_{t}\left(\hat{E}_{u}^{-1 / 2}\right)^{\prime},
$$

where $\hat{E}_{u}=(1 / T) \sum_{t=1}^{T} \hat{U}_{t}$. The rescaled $\tilde{U}_{t}$ can then be used to generate bootstrap replicates of $C_{T+j}$, for $j=1, \ldots, h$, where $h$ denotes the chosen forecast horizon.

Step 3 Draw with replacement a bootstrap sample $\left\{\tilde{U}_{T+1 \mid T}, \ldots, \tilde{U}_{T+h \mid T}\right\}$ of length $h$ from the empirical CDF of $\left\{\tilde{U}_{t}, t=1, \ldots, T\right\}$.

Step 4 For $j=1 \ldots, h$, recursively generate a sequence of bootstrap replicates of $C_{T+j}$ as follows

$$
\begin{aligned}
M_{T+j \mid T} & =\hat{\Lambda}(\theta, \omega)+\hat{\theta} \sum_{k=1}^{K} \phi_{k}(\hat{\omega}) \tilde{C}_{T-k+j \mid T} \\
L_{T+j \mid T} & =M_{T+j \mid T}^{1 / 2} \\
C_{T+j \mid T}^{*} & =L_{T+j \mid T} C_{T+j}\left(L_{T+j \mid T}^{\prime}\right)^{-1} \\
P_{T+j \mid T}^{*} & =\left(\operatorname{diag}\left\{C_{T+j \mid T}^{*}\right\}\right)^{-1 / 2} C_{T+j \mid T}^{*}\left(\operatorname{diag}\left\{C_{T+j \mid T}^{*}\right\}\right)^{-1 / 2} \\
S_{i i, T+j \mid T}^{*} & =\left(1-\hat{\gamma}_{i}-\hat{\delta}_{i}\right)+\hat{\gamma}_{i} C_{i i, T+j-1 \mid T}^{*}+\hat{\delta}_{i} S_{i i, T+j-1 \mid T}^{*} \\
R_{T+j \mid T}^{*} & =(1-\hat{\alpha}-\hat{\beta}) I_{n}+\hat{\alpha} P_{T+j-1 \mid T}^{*}+\hat{\beta} R_{T+j-1 \mid T}^{*} \\
S_{T+j \mid T}^{*} & =\left(\operatorname{diag}\left\{S_{T+j \mid T}^{*}\right\}\right)^{1 / 2} R_{T+j \mid T}^{*}\left(\operatorname{diag}\left\{S_{T+j \mid T}^{*}\right\}\right)^{1 / 2} \\
S_{T+j \mid T} & =L_{T+j \mid T} S_{T+j \mid T}^{*} L_{T+j \mid T}^{\prime} \\
C_{T+j \mid T}= & =\left(S_{T+j \mid T}^{1 / 2}\right) \tilde{U}_{T+j}\left(S_{T+j \mid T}^{1 / 2}\right)^{\prime}
\end{aligned}
$$

Step 5 Repeat steps 3-4 B times, where B is set sufficiently large (e.g. $\mathrm{B}=10000)$. As a result the procedure generates an array of $h \times B$ bootstrap replicates $C_{T+j \mid T}^{(b)}(b=1, \ldots, B)$. Step 6 Finally, the h-steps-ahead forecast can be computed as

$$
\hat{S}_{T, j}=\frac{1}{B} \sum_{b=1}^{B} C_{T+j \mid T}^{(b)}
$$

Even if our primary interest is in forecasting from MMReDCC models, the proposed forecasting procedure is very general and can be readily adapted to any model that admits the representation in Eq.(9), where $S_{t}$ is modeled as a function of past information $\mathcal{I}_{t-1}$. For example, in the empirical application which is being presented in Section 6.2, we also use it to generate multi-step ahead forecasts of $C_{t}$ from the cRDCC model of Bauwens et al. [7]. To this purpose, the dynamic equations in step 4 must be replaced by those pertaining to the specific model of interest. 


\section{Empirical Applications}

This section contains two empirical applications. The first application provides the estimation results for the IMP estimator in comparison with the standard QML estimator in the ideal case where both can be computed. The second one is performed in a large dimensional system and aims at evaluating both the full-sample fit of the model and its forecasting performance. Specifically, we evaluate the ability of the MMReDCC model to provide accurate multi-step-ahead covariance predictions against existing competitors not accounting for time-varying long term dynamics.

\subsection{Small sample accuracy comparison}

As regards the in-sample performance, we are interested in comparing the estimates provided by the IMP method to the QML ones that are obtained by maximizing the likelihood over the full parameter vector and can only be used in low dimensional cases (remember Table 1). For this purpose, we fix the cross-sectional dimension equal to ten assets and use three different datasets. An overview of the data being used is given in Appendix A. The first dataset comprises the assets used in Bauwens et al. [5] and includes series of daily realized covariance matrices estimated on five minute intraday returns over the period February 2001 to December 2009; the second and third sets comprise arbitrary selected subsamples of the dataset used in the work of Boudt et al. [10] which consists of series of daily realized covariance matrices obtained with the CholCov estimator over the period January 2007 to December 2012. ${ }^{3}$ As already mentioned before, the choice of the realized estimator is not an issue here as the model can be fitted to any series of realized variance-covariance matrices as long as they are guaranteed to be PDS.

Estimation results for the MMReDCC model by both the IMP and the QML estimators are reported in Table 6 .

In the three datasets considered, both methods appear to deliver similar estimates. Short term GARCH coefficients tend to be quite homogeneous across assets and generally significant; the same applies to the short term correlation estimates. As for the parameters driving the long term component, it can be noticed that the estimated $\theta$ and $\omega$ coefficients are regularly lower for the IMP than for the QML method. This is in line with the prevailing negative bias reported from the simulation study. The QML estimator, as expected, performs slightly better than the IMP but the small differences in the log-likelihood values indicate that the loss, in terms of goodness of fit, is negligible and that the proposed IMP algorithm represents an acceptable approach even when the model cannot be estimated by QML.

\footnotetext{
${ }^{3}$ Our analysis focuses on open-to-close covariance matrices, whereby noisy overnight returns have not been included in the construction of the estimators. We refer to the cited papers for further details.
} 
Table 6: Application I. In-sample parameter estimates

\begin{tabular}{|c|c|c|c|}
\hline \multicolumn{4}{|c|}{ Dataset 1} \\
\hline \multicolumn{2}{|c|}{ QML } & \multicolumn{2}{|c|}{ IMPL } \\
\hline$\gamma_{i}$ & $\delta_{i}$ & $\gamma_{i}$ & $\delta_{i}$ \\
\hline $\begin{array}{l}0.36 \\
(0.04)\end{array}$ & $\begin{array}{l}0.55 \\
(0.05)\end{array}$ & $\begin{array}{l}0.38 \\
(0.06)\end{array}$ & $\begin{array}{l}0.56 \\
(0.04)\end{array}$ \\
\hline $\begin{array}{l}0.35 \\
(0.63)\end{array}$ & $\begin{array}{c}0.59 \\
(0.03)\end{array}$ & $\underset{(0.07)}{0.41}$ & $\begin{array}{l}0.51 \\
(0.04)\end{array}$ \\
\hline $\begin{array}{l}0.34 \\
(0.04)\end{array}$ & $\begin{array}{l}0.57 \\
(0.06)\end{array}$ & $\begin{array}{l}0.37 \\
(0.05)\end{array}$ & $\begin{array}{l}0.53 \\
(0.06)\end{array}$ \\
\hline $\begin{array}{l}0.33 \\
(0.04)\end{array}$ & $\begin{array}{l}0.60 \\
(0.06)\end{array}$ & $\begin{array}{l}0.31 \\
(0.04)\end{array}$ & $\begin{array}{l}0.59 \\
(0.05)\end{array}$ \\
\hline $\begin{array}{l}0.34 \\
(0.03)\end{array}$ & $\begin{array}{l}0.64 \\
(0.05)\end{array}$ & $\begin{array}{l}0.33 \\
(0.04)\end{array}$ & $\begin{array}{l}0.60 \\
(0.04)\end{array}$ \\
\hline $\begin{array}{l}0.26 \\
(0.03)\end{array}$ & $\begin{array}{l}0.64 \\
(0.05)\end{array}$ & $\begin{array}{l}0.25 \\
(0.03)\end{array}$ & $\begin{array}{l}0.66 \\
(0.04)\end{array}$ \\
\hline $\begin{array}{l}0.38 \\
(0.03)\end{array}$ & $\begin{array}{l}0.51 \\
(0.05)\end{array}$ & $\begin{array}{l}0.37 \\
(0.03)\end{array}$ & $\begin{array}{l}0.51 \\
(0.03)\end{array}$ \\
\hline $\begin{array}{l}0.38 \\
(0.04)\end{array}$ & $\begin{array}{l}0.51 \\
(0.05)\end{array}$ & $\begin{array}{l}0.37 \\
(0.03)\end{array}$ & $\begin{array}{l}0.52 \\
(0.04)\end{array}$ \\
\hline $\begin{array}{l}0.37 \\
(0.04)\end{array}$ & $\begin{array}{l}0.52 \\
(0.05)\end{array}$ & $\begin{array}{l}0.39 \\
(0.04)\end{array}$ & $\begin{array}{l}0.52 \\
(0.04)\end{array}$ \\
\hline $\begin{array}{l}0.36 \\
(0.04) \\
\end{array}$ & $\begin{array}{l}0.50 \\
(0.04) \\
\end{array}$ & $\begin{array}{l}0.37 \\
(0.04) \\
\end{array}$ & $\begin{array}{l}0.53 \\
(0.05) \\
\end{array}$ \\
\hline$\alpha$ & $\beta$ & $\alpha$ & $\beta$ \\
\hline $\begin{array}{l}0.07 \\
(0.00) \\
\end{array}$ & $\begin{array}{l}0.84 \\
(0.01) \\
\end{array}$ & $\begin{array}{l}0.07 \\
(0.00) \\
\end{array}$ & $\begin{array}{l}0.88 \\
(0.00) \\
\end{array}$ \\
\hline$\theta$ & $\omega$ & $\theta$ & $\omega$ \\
\hline $\begin{array}{l}0.91 \\
(0.05) \\
\end{array}$ & $\begin{array}{l}4.56 \\
(0.06) \\
\end{array}$ & $\begin{array}{l}0.88 \\
(0.05) \\
\end{array}$ & $\begin{array}{l}4.52 \\
(0.05) \\
\end{array}$ \\
\hline
\end{tabular}

Frobenius Norm

\begin{tabular}{cc}
\multicolumn{2}{c}{1.60} \\
\hline Loglik & Loglik \\
-18317 & -18323 \\
\hline
\end{tabular}

Dataset 2

\begin{tabular}{|c|c|c|c|}
\hline \multicolumn{2}{|c|}{ QML } & \multicolumn{2}{|c|}{ IMP } \\
\hline$\gamma_{i}$ & $\delta_{i}$ & $\gamma_{i}$ & $\delta_{i}$ \\
\hline $\begin{array}{l}0.15 \\
(0.03)\end{array}$ & $\begin{array}{l}0.75 \\
(0.06)\end{array}$ & $\begin{array}{l}0.18 \\
(0.05)\end{array}$ & $\begin{array}{l}0.77 \\
(0.05)\end{array}$ \\
\hline $\begin{array}{l}0.23 \\
(0.05)\end{array}$ & $\begin{array}{l}0.69 \\
(0.07)\end{array}$ & $\begin{array}{l}0.23 \\
(0.05)\end{array}$ & $\begin{array}{l}0.71 \\
(0.06)\end{array}$ \\
\hline $\begin{array}{l}0.24 \\
(0.06)\end{array}$ & $\begin{array}{l}0.61 \\
(0.09)\end{array}$ & $\begin{array}{l}0.26 \\
(0.06)\end{array}$ & $\begin{array}{l}0.64 \\
(0.07)\end{array}$ \\
\hline $\begin{array}{l}0.20 \\
(0.28)\end{array}$ & $\begin{array}{l}0.65 \\
(0.41)\end{array}$ & $\begin{array}{l}0.19 \\
(0.08)\end{array}$ & $\begin{array}{l}0.73 \\
(0.09)\end{array}$ \\
\hline $\begin{array}{c}0.22 \\
(0.1)\end{array}$ & $\begin{array}{l}0.49 \\
(0.06)\end{array}$ & $\begin{array}{l}0.19 \\
(0.08)\end{array}$ & $\begin{array}{l}0.72 \\
(0.10)\end{array}$ \\
\hline $\begin{array}{l}0.18 \\
(0.09)\end{array}$ & $\begin{array}{l}0.67 \\
(0.12)\end{array}$ & $\begin{array}{l}0.18 \\
(0.06)\end{array}$ & $\begin{array}{l}0.76 \\
(0.08)\end{array}$ \\
\hline $\begin{array}{c}0.18 \\
(0.2)\end{array}$ & $\begin{array}{l}0.56 \\
(0.09)\end{array}$ & $\begin{array}{l}0.08 \\
(0.04)\end{array}$ & $\begin{array}{l}0.89 \\
(0.05)\end{array}$ \\
\hline $\begin{array}{l}0.18 \\
(0.09)\end{array}$ & $\begin{array}{l}0.75 \\
(0.09)\end{array}$ & $\begin{array}{l}0.18 \\
(0.04)\end{array}$ & $\begin{array}{l}0.76 \\
(0.04)\end{array}$ \\
\hline $\begin{array}{l}0.18 \\
(0.06)\end{array}$ & $\begin{array}{l}0.73 \\
(0.08)\end{array}$ & $\begin{array}{l}0.18 \\
(0.05)\end{array}$ & $\begin{array}{l}0.76 \\
(0.05)\end{array}$ \\
\hline $\begin{array}{l}0.14 \\
(0.04) \\
\end{array}$ & $\begin{array}{c}0.77 \\
(0.08) \\
\end{array}$ & $\begin{array}{l}0.13 \\
(0.04) \\
\end{array}$ & $\begin{array}{l}0.83 \\
(0.05) \\
\end{array}$ \\
\hline$\alpha$ & $\beta$ & $\alpha$ & $\beta$ \\
\hline $\begin{array}{c}0.017 \\
(0.00)\end{array}$ & $\begin{array}{c}0.80 \\
(0.1)\end{array}$ & $\begin{array}{c}0.018 \\
(0.00)\end{array}$ & $\begin{array}{l}0.91 \\
(0.05)\end{array}$ \\
\hline$\theta$ & $\omega$ & $\theta$ & $\omega$ \\
\hline $\begin{array}{l}0.84 \\
(0.18)\end{array}$ & $\begin{array}{l}5.63 \\
(2.4)\end{array}$ & $\begin{array}{l}0.81 \\
(0.18)\end{array}$ & $\begin{array}{l}3.60 \\
(0.65)\end{array}$ \\
\hline
\end{tabular}

Frobenius Norm

\begin{tabular}{cc}
\multicolumn{2}{c}{3.83} \\
\hline Loglik & Loglik \\
-28571 & -28576 \\
\hline
\end{tabular}

Dataset 3

\begin{tabular}{cccc}
\multicolumn{2}{c}{ QML } & \multicolumn{2}{c}{ IMP } \\
\hline$\gamma_{i}$ & $\delta_{i}$ & $\gamma_{i}$ & $\delta_{i}$ \\
\hline 0.20 & 0.73 & 0.18 & 0.78 \\
$(0.05)$ & $(0.12)$ & $(0.07)$ & $(0.08)$ \\
0.21 & 0.70 & 0.22 & 0.72 \\
$(0.06)$ & $(0.23)$ & $(0.04)$ & $(0.05)$ \\
0.25 & 0.66 & 0.22 & 0.69 \\
$(0.05)$ & $(0.07)$ & $(0.06)$ & $(0.06)$ \\
0.12 & 0.79 & 0.05 & 0.93 \\
$(0.07)$ & $(0.20)$ & $(0.01)$ & $(0.02)$ \\
0.18 & 0.76 & 0.16 & 0.79 \\
$(0.14)$ & $(0.41)$ & $(0.03)$ & $(0.04)$ \\
0.29 & 0.58 & 0.24 & 0.67 \\
$(0.10)$ & $(0.17)$ & $(0.07)$ & $(0.09)$ \\
0.14 & 0.80 & 0.20 & 0.59 \\
$(0.05)$ & $(0.50)$ & $(0.13)$ & $(0.15)$ \\
0.21 & 0.71 & 0.22 & 0.71 \\
$(0.10)$ & $(0.11)$ & $(0.06)$ & $(0.07)$ \\
0.22 & 0.73 & 0.17 & 0.76 \\
$(0.06)$ & $(0.21)$ & $(0.10)$ & $(0.09)$ \\
0.15 & 0.69 & 0.02 & 0.74 \\
$(0.06)$ & $(0.10)$ & $(0.08)$ & $(0.03)$ \\
\hline$\alpha$ & $\beta$ & $\alpha$ & $\beta$ \\
\hline 0.02 & 0.96 & 0.01 & 0.90 \\
$(0.00)$ & $(0.01)$ & $(0.00)$ & $(0.04)$ \\
\hline$\theta$ & $\omega$ & $\theta$ & $\omega$ \\
\hline 0.90 & 3.46 & 0.80 & 2.67 \\
$(0.14)$ & $(0.71)$ & $(0.22)$ & $(0.51)$ \\
\hline
\end{tabular}

Frobenius Norm

\begin{tabular}{cc}
\multicolumn{2}{c}{2.81} \\
\hline Loglik & Loglik \\
-29026 & -29056 \\
\hline
\end{tabular}

Note: Each panel reports parameter estimates and corresponding standard errors in brackets. The Frobenius norm measures the difference between the two long term MIDAS intercepts and is computed as $\sqrt{\sum_{i, j=1}^{n} \mid \hat{\Lambda}_{i, j}^{Q M L}-\hat{\Lambda}_{i, j}^{\left.I M P\right|^{2}}}$, where the superscripts $Q M L, I M P$ denote respectively the one-step quasi-maximum likelihood and the iterative profile likelihood estimators. Number of in-sample observations is 2242 for Dataset 1 and 1499 for Dataset 2 and 3.

\subsection{Forecasting performance}

In this subsection we push the analysis to higher dimensions, with the aim of assessing the usefulness of the MMReDCC model in a forecasting framework. As benchmarks we consider the Consistent RDCC (cRDCC) model of Bauwens et al. [7] as the closest competitor and a simple Exponentially Weighted Moving Average (EWMA) model. The simple EWMA predictor appears a natural candidate due to its widespread diffusion among practitioners and in risk management systems like RiskMetrics. If applied to the realized 
covariance matrices, it is defined by

$$
S_{t}=(1-\lambda) C_{t-1}+\lambda S_{t-1}
$$

where the $\lambda$ parameter is set equal to the value 0.94 (see also Golosnoy et al. [21]).

On the other hand, the choice of the cRDCC as a benchmark is supported by two main reasons. First, it assumes that conditional volatilities and correlations mean revert to constant quantities, thus it can be considered as a simplified version of the MMReDCC model despite not being formally nested in it. Second, the findings of Boudt et al. [10] show that the cRDCC model favorably compares with some widely used competitors, such as the HEAVY (Noureldin et al. [28]) and the cDCC (Aielli [1]) model, in forecasting Value-at-Risk. In order to estimate the cRDCC in high dimension, we apply a three stage QML estimation procedure as suggested by Bauwens et al. [7], where the constant long term covariance matrix is consistently targeted by the unconditional covariance. This drastically reduces the number of parameters to be estimated to $2 n+2$.

The dataset comprises fifty of the most liquid equities of the S\&P 500 traded over the period May 1997 - July 2008, for a total of 2524 observations. Tickers and descriptive statistics of the data are given in Appendix B.

Before turning to the out-of-sample analysis, it is worth first looking at the estimates obtained by fitting the MMReDCC and cRDCC models over the full sample period. As emerges from Panel A of Table 7, the MMReDCC outperforms the cRDCC in terms of the AIC and BIC criteria, which are both minimized for the MMReDCC. The univariate $\operatorname{GARCH}(1,1)$ parameters $\bar{\gamma}$ and $\bar{\delta}$, reported in averaged values across series, largely agree with each other, while the correlation estimates are markedly different across the two models.

To closely examine the difference between the MMReDCC and cRDCC models, consider the conditional correlations between two selected stocks, APOL and GCI, presented in Figure 1. The parameter estimates from the MMReDCC produce large and more persistent shifts in the conditional correlations, including a marked increase at the beginning of May, 2007, lasting until the end of the sample. The cRDCC model, on the contrary, delivers conditional correlations that are nearly constant and exhibit little variation even near the spread of the financial crisis events in 2008. Given the close similarity between the models, this can be reasonably explained by the fact that the parameters $\theta$ and $\omega$ driving the long term (co)volatilities dynamics allow much more flexibility of the MMReDCC model and thus for a better responsiveness of correlations in periods of higher market volatility.

To determine whether the MMReDCC model can lead to forecasting gains we compute forecasts of the conditional covariance matrix of daily returns at different horizons making use of the bootstrap procedure explained in Section 5. A similar approach is also applied to the cRDCC model, while predictions from the EWMA are obtained analytically, since this model implies that $E\left(C_{t+h} \mid \Im_{t}\right)=E\left(C_{t+h-1} \mid \Im_{t}\right)$.

To shorten the computational time, estimation is performed using a fixed-rolling window scheme with window length equal to 2024 observations, that shifts forward every twenty days, over which parameter estimates are kept fixed. Given the full sample size of 2524 
Table 7: Full sample estimates and Implemented loss functions.

\begin{tabular}{lcc}
\hline \multicolumn{3}{l}{ Panel A: Full sample estimates } \\
\hline $\bar{\gamma}$ & 0.396 & cRDCC \\
$\bar{\delta}$ & 0.601 & 0.351 \\
$\alpha$ & 0.013 & 0.592 \\
$\beta$ & 0.951 & 0.027 \\
$\theta$ & 0.719 & 0.875 \\
$\omega$ & 2.068 & \\
\hline Lik & 800141 & -577064 \\
AIC & -632 & -599 \\
BIC & -629 & \\
\hline Panel B: Implemented Loss functions & \\
\hline Frob & Frobenius distance & $\operatorname{tr}\left[\left(C_{t}-H_{t}\right)^{\prime}\left(C_{t}-H_{t}\right)\right]$ \\
Sfrob & Squared Frobenius distance & $\sum_{i=1}^{n} \lambda_{i}$ \\
Euclid & Euclidean distance & $\operatorname{vech}\left(C_{t}-H_{t}\right) / \mathrm{vech}\left(C_{t}-H_{t}\right)$ \\
ST & Stein & $\operatorname{tr}\left(H_{t}^{-1} C_{t}\right)-\log \left|H_{t}^{-1} C_{t}\right|-n$ \\
vND & von Neumann Divergence & $\operatorname{tr}\left(C_{t} \log C_{t}-C_{t} \log H_{t}-C_{t}+H_{t}\right)$ \\
QLIK & Qlike & $\log \left|H_{t}\right|+\operatorname{tr}\left(H_{t}^{-1} C_{t}\right)$ \\
\hline
\end{tabular}

Note: Panel A reports full sample estimates from the MMReDCC and cRDCC model, with AIC and BIC criteria rescaled by the number of observations. Panel B contains the loss functions chosen to evaluate the models forecasting ability. $H_{t}$ denotes the predicted conditional covariance matrix while $C_{t}$ is the realized measure; $\lambda_{i}$ are the eigenvalues of $\left(C_{t}-H_{t}\right)^{2}$ and $n$ denotes the number of assets.

observations, this leads to a number of re-estimations of each model equal to twenty-five. The out-of-sample period starts on July, 2006 and covers roughly the last 500 observations of the sample, ending just before the heat of the financial crisis (July 2008). The horizons considered for predictions are $h=1,5,10$ and 20 days.

The comparison of the models forecasting ability is performed using the six consistent ${ }^{4}$ loss functions defined in Panel B of Table 7, for which we report averaged values over the out-of-sample period. In order to evaluate the significance of the loss function differences we employ the model confidence set (MCS) approach of Hansen et al. [23], which identifies the single model or the set of models having the best forecasting performance at a given confidence level. ${ }^{5}$

Results are summarized by horizon in Table 8. According to Panel A, at the shortest horizon the MMReDCC model is outperformed by its competitors on all the selected criteria, and excluded by the MCS at both the 90 and $75 \%$ confidence levels. This suggests that

\footnotetext{
${ }^{4}$ The term consistent is used according to Laurent et al. [26].

${ }^{5}$ The MCS is computed at the $90 \%$ and $75 \%$ confidence levels, with block-length bootstrap parameter and number of bootstrap samples used to obtain the distribution under the null respectively equal to 2 and 10000 .
} 
Figure 1: Estimated correlation of APOL-GCI.

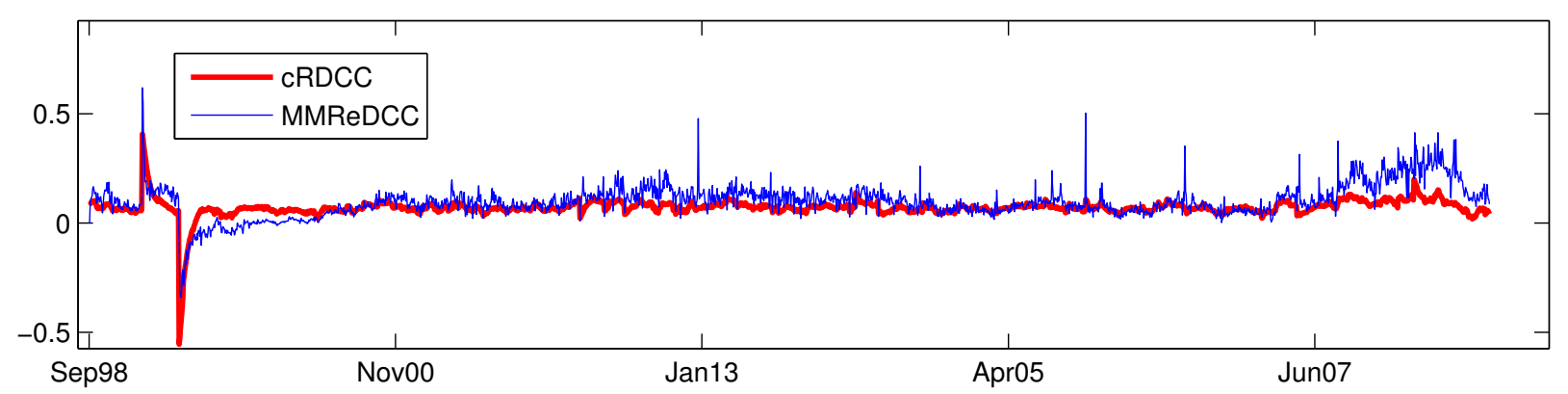

for the data at hand, accurate one-step-ahead predictions can be obtained by employing simpler models that do not necessarily account for a time-varying long run level. ${ }^{6}$ At this stage the choice between the EWMA and the cRDCC model appears almost indifferent, despite the latter being more often included in the MCS.

As we move further in time the situation is quickly reversed: at the 5-day horizon, the MMReDCC model minimizes five out of six loss functions while at the 10-day horizon it appears to deliver the optimal covariance forecasts according to the whole set of losses. This gain is confirmed by the inclusion of the model in the MCS resulting from all the selected criteria, differently from the competitors which are almost never included (EWMA and cRDCC are included three and two times, respectively, in the $75 \%$ MCS at $h=5$, but never at $h=10)$. The predominance of the MMReDCC remains quite stable even at the longest horizon $(h=20)$, but the difference in the forecast accuracy between the MMReDCC and the benchmarks becomes smaller, with the cRDCC performing almost as good as the MMReDCC in terms of MCS inclusions (cRDCC excluded only in the von Neumann $75 \%$ MCS). These results appear to be in line with those of [21], where already at the 10-ahead horizon the differences in the forecast accuracy between their best component CAW model and the selected benchmarks were smaller than at shorter horizons.

Overall, the out-of-sample performance of the MMReDCC model in a moderately volatile time period appears to be good relative to the competing models especially at medium-term horizons, when it yields the most accurate forecasts. In light of these empirical results, it appears that the introduction of an additional component capturing the secular movements in the volatility and covolatility dynamics is well justified and useful to enhance a higher forecasting accuracy.

\footnotetext{
${ }^{6}$ This result is quite different from that of Bauwens et al. [5] where models with a time-varying long-run component dominate models with a constant long-run level at forecast horizon 1 . The dataset of that paper covers the turbulent period of 2008 and 2009.
} 
Table 8: Multi-step-ahead forecast evaluation

\begin{tabular}{|c|c|c|c|c|c|c|c|c|c|c|c|c|}
\hline & \multicolumn{3}{|c|}{ Horizon 1} & \multicolumn{3}{|c|}{ Horizon 5} & \multicolumn{3}{|c|}{ Horizon 10} & \multicolumn{3}{|c|}{ Horizon 20} \\
\hline & MMReDCC & EWMA & $\mathrm{cRDCC}$ & MMReDCC & EWMA & cRDCC & MMReDCC & EWMA & $\mathrm{cRDCC}$ & MMReDCC & EWMA & $\mathrm{cRDCC}$ \\
\hline \multicolumn{13}{|c|}{ Panel A: Loss functions } \\
\hline Frob & 0.133 & 0.125 & 0.126 & 0.138 & 0.135 & 0.139 & 0.144 & 0.148 & 0.148 & 0.158 & 0.167 & 0.160 \\
\hline Sfrob & 0.040 & 0.034 & 0.037 & 0.036 & 0.036 & 0.036 & 0.037 & 0.038 & 0.038 & 0.041 & 0.042 & 0.043 \\
\hline Euclid & 0.086 & 0.080 & 0.082 & 0.087 & 0.088 & 0.088 & 0.091 & 0.093 & 0.093 & 0.099 & 0.102 & 0.101 \\
\hline ST & 65.159 & 59.018 & 55.449 & 59.227 & 61.684 & 60.245 & 61.522 & 63.611 & 63.289 & 67.748 & 66.269 & 66.667 \\
\hline vND & 0.018 & 0.017 & 0.017 & 0.018 & 0.019 & 0.019 & 0.019 & 0.022 & 0.020 & 0.022 & 0.025 & 0.022 \\
\hline QLIK & -370.64 & -376.78 & -380.35 & -376.66 & -374.20 & -375.64 & -374.43 & -372.34 & -372.67 & -368.07 & -369.55 & -369.15 \\
\hline \multicolumn{13}{|c|}{ Panel B: $90 \% M C S$} \\
\hline Frob & 0.014 & 0.689 & 1.000 & 0.486 & 1.000 & 0.232 & 1.000 & 0.001 & 0.015 & 0.224 & 0.619 & 1.000 \\
\hline Sfrob & 0.044 & 0.259 & 1.000 & 0.614 & 1.000 & 0.614 & 1.000 & 0.052 & 0.052 & 0.547 & 0.547 & 1.000 \\
\hline Euclid & 0.023 & 0.464 & 1.000 & 1.000 & 0.311 & 0.420 & 1.000 & 0.001 & 0.016 & 0.506 & 0.506 & 1.000 \\
\hline ST & 0.000 & 0.000 & 1.000 & 1.000 & 0.000 & 0.018 & 1.000 & 0.000 & 0.003 & 1.000 & 0.000 & 0.281 \\
\hline vND & 0.000 & 0.263 & 1.000 & 1.000 & 0.002 & 0.028 & 1.000 & 0.000 & 0.000 & 1.000 & 0.000 & 0.159 \\
\hline QLIK & 0.000 & 0.000 & 1.000 & 1.000 & 0.000 & 0.017 & 1.000 & 0.000 & 0.004 & 1.000 & 0.000 & 0.275 \\
\hline \multicolumn{13}{|c|}{ Panel C: $75 \%$ MCS } \\
\hline Frob & 0.013 & 0.695 & 1.000 & 0.497 & 1.000 & 0.227 & 1.000 & 0.003 & 0.016 & 1.000 & 0.218 & 0.615 \\
\hline Sfrob & 0.047 & 1.000 & 0.268 & 0.631 & 1.000 & 0.631 & 1.000 & 0.051 & 0.051 & 1.000 & 0.545 & 0.545 \\
\hline Euclid & 0.018 & 0.461 & 1.000 & 1.000 & 0.315 & 0.429 & 1.000 & 0.001 & 0.015 & 1.000 & 0.511 & 0.511 \\
\hline $\mathrm{ST}$ & 0.000 & 0.000 & 1.000 & 1.000 & 0.000 & 0.015 & 1.000 & 0.000 & 0.003 & 1.000 & 0.000 & 0.278 \\
\hline vND & 0.000 & 0.265 & 1.000 & 1.000 & 0.002 & 0.029 & 1.000 & 0.000 & 0.000 & 1.000 & 0.000 & 0.160 \\
\hline QLIK & 0.000 & 0.000 & 1.000 & 1.000 & 0.000 & 0.014 & 1.000 & 0.000 & 0.003 & 1.000 & 0.000 & 0.274 \\
\hline
\end{tabular}

Note: Panel A reports averaged values of the loss functions listed in Table 7 over the out-of-sample period, where the best performing model within each row is in bold. Entries in Panel B and C are p-values of the MCS with $10 \%$ and $25 \%$ size, respectively. Included models in bold.

\section{Conclusions}

The estimation procedure proposed in the paper allows to extend the range of applicability of the MMReDCC model to large dimensional portfolios such as those encountered in standard risk management practice. In order to reach this objective, we face two wellknown challenges in multivariate time series modeling, namely high-dimensional estimation and multi-step ahead forecasting.

To face the former challenge, we implement a feasible estimation procedure, the Iterative Moment based Profiling (IMP) algorithm. It profiles out the parameters of the scale MIDAS intercept matrix and iteratively maximizes the likelihood in terms of the other parameters of interest. Whilst not providing an asymptotic inference theory for this method, we investigate the finite sample properties of the estimator via a simulation study, which demonstrates that the IMP estimator is comparable to the one-step QML ones in terms of bias and accuracy. We also compare the one-step QML estimator with the IMP estimator on real data sets of small dimension (ten) and find that not only the two estimators deliver very similar in-sample estimates, but also the loss of the IMP in terms of likelihood values can be considered as negligible. Another application illustrates the usefulness of the IMP algorithm when the model is applied to the realized covariances of fifty stocks. From the 
computational point of view, the IMP algorithm is found to be reliable and easy to apply despite the large number of parameters involved in the MMReDCC model. Given its flexibility, we fairly believe that it could be applied to datasets of larger dimensions.

As regards the second challenge, we develop a bootstrap approach to the generation of multi-step-ahead predictions. In an application to a portfolio of fifty stocks, we provide compelling evidence that the MMReDCC model is useful for out-of-sample forecasting purposes even when one has to work in such a dimension. If compared with existing multivariate competitors not accounting for time-varying long-term dynamics, the MMReDCC is found to deliver the most accurate predictions especially at medium-term horizons, thus indicating the importance of allowing for a long-run component. 


\section{Appendix A. Application I: Descriptive statistics of daily realized variances.}

\begin{tabular}{|c|c|c|c|c|c|c|c|}
\hline Symbol & Issue name & Mean & Max. & Min. & Std.dev. & Skewness & Kurtosis \\
\hline & \multicolumn{5}{|c|}{ Dataset 1: February, 2001 - December, 2009} & & \\
\hline AA & Alcoa & 5.458 & 277.308 & 0.074 & 16.811 & 7.178 & 72.570 \\
\hline AXP & American Express & 5.055 & 176.478 & 0.112 & 11.094 & 7.529 & 84.686 \\
\hline $\mathrm{BAC}$ & Bank of America & 1.934 & 57.543 & 0.075 & 3.362 & 7.319 & 85.006 \\
\hline KO & Coca Cola & 2.455 & 43.106 & 0.084 & 3.412 & 4.724 & 36.234 \\
\hline DD & Du Pont & 2.073 & 115.378 & 0.126 & 4.155 & 13.296 & 288.066 \\
\hline $\mathrm{GE}$ & General Electric & 4.944 & 160.241 & 0.294 & 8.935 & 7.635 & 92.124 \\
\hline IBM & International Business Machines & 4.420 & 201.879 & 0.077 & 9.154 & 8.536 & 133.699 \\
\hline JPM & JP Morgan & 2.529 & 63.874 & 0.163 & 3.728 & 6.442 & 68.505 \\
\hline MSFT & Microsoft & 3.196 & 114.256 & 0.097 & 7.114 & 7.232 & 75.484 \\
\hline \multirow[t]{2}{*}{ XOM } & Exxon Mobil & 1.414 & 56.505 & 0.039 & 2.254 & 9.715 & 180.206 \\
\hline & \multicolumn{5}{|c|}{ Dataset 2: January, 2007 - December, 2012} & & \\
\hline ACAS & American Capital & 8.576 & 331.786 & 0.060 & 20.844 & 7.226 & 78.667 \\
\hline AET & Aetna & 8.163 & 771.525 & 0.109 & 26.593 & 17.882 & 467.969 \\
\hline AFL & Aflac Incorporated & 9.113 & 675.348 & 0.133 & 27.345 & 13.811 & 284.791 \\
\hline AIG & American International Group & 8.799 & 555.098 & 0.103 & 26.382 & 11.459 & 185.778 \\
\hline AIZ & Assurant & 8.613 & 325.167 & 0.101 & 23.230 & 7.712 & 79.082 \\
\hline ALL & The Allstate Coprporation & 8.213 & 543.714 & 0.186 & 24.593 & 11.277 & 189.052 \\
\hline AMP & Ameriprise Financial & 7.679 & 264.761 & 0.129 & 17.790 & 6.098 & 54.262 \\
\hline AXP & American Express Company & 8.076 & 945.750 & 0.095 & 30.571 & 21.795 & 618.891 \\
\hline BAC & Bank of America & 8.450 & 332.586 & 0.130 & 22.830 & 8.458 & 96.824 \\
\hline \multirow[t]{2}{*}{ BBT } & BB\&T Corporation & 9.093 & 613.826 & 0.087 & 28.801 & 11.267 & 184.837 \\
\hline & \multicolumn{5}{|c|}{ Dataset 3: January, 2007 - December, 2012} & & \\
\hline STI & SunTrust Banks & 8.510 & 388.707 & 0.086 & 22.765 & 8.088 & 94.838 \\
\hline STT & State Street Corporation & 8.985 & 315.656 & 0.039 & 23.192 & 7.265 & 74.391 \\
\hline TMK & Torchmark Corporation & 8.748 & 537.273 & 0.022 & 24.479 & 10.543 & 176.515 \\
\hline TROW & T.Rowe Price Group & 8.991 & 425.263 & 0.073 & 25.019 & 8.753 & 108.221 \\
\hline UNH & UnitedHealth Group & 8.344 & 378.667 & 0.130 & 21.065 & 8.534 & 112.479 \\
\hline UNM & Unun Group & 8.046 & 309.086 & 0.044 & 19.440 & 8.564 & 107.272 \\
\hline USB & U.S.Bancorp & 10.176 & 2534.073 & 0.079 & 69.496 & 32.392 & 1164.053 \\
\hline WFC & Wells Fargo \& Company & 9.481 & 525.034 & 0.106 & 30.162 & 10.717 & 156.791 \\
\hline WU & The Western Union Company & 8.775 & 484.124 & 0.056 & 25.204 & 9.954 & 145.509 \\
\hline ZION & Zions Bancorporation & 15.302 & 6855.823 & 0.136 & 197.794 & 30.674 & 1005.621 \\
\hline
\end{tabular}


Appendix B. Application II: descriptive statistics of daily realized variances.

\begin{tabular}{|c|c|c|c|c|c|c|}
\hline \multirow[b]{2}{*}{ Stock } & \multicolumn{6}{|c|}{ Estimation sample: May 12, 1997 to July 17,2006 (2024 observations) } \\
\hline & Mean $(\mathrm{e}-03)$ & Max. & Min.(e-03) & Std.dev.(e-03) & Skewness & Kurtosis \\
\hline ABT & 0.434 & 0.025 & 0.026 & 0.948 & 15.958 & 333.606 \\
\hline AFL & 0.491 & 0.027 & 0.020 & 1.208 & 13.097 & 225.997 \\
\hline APD & 0.490 & 0.075 & 0.020 & 1.896 & 32.234 & 1221.466 \\
\hline $\mathrm{AA}$ & 0.540 & 0.012 & 0.040 & 0.759 & 7.886 & 94.971 \\
\hline ALL & 0.497 & 0.098 & 0.012 & 2.319 & 37.311 & 1554.460 \\
\hline AXP & 0.549 & 0.041 & 0.014 & 1.553 & 16.921 & 374.949 \\
\hline AIG & 0.391 & 0.073 & 0.027 & 1.724 & 38.011 & 1597.332 \\
\hline ADI & 1.375 & 0.043 & 0.070 & 2.153 & 7.635 & 103.080 \\
\hline $\mathrm{APOL}$ & 1.276 & 0.080 & 0.043 & 2.556 & 16.984 & 470.125 \\
\hline T & 0.620 & 0.046 & 0.017 & 1.659 & 15.676 & 339.927 \\
\hline AZO & 0.497 & 0.046 & 0.021 & 1.250 & 25.934 & 909.714 \\
\hline $\mathrm{AVY}$ & 0.416 & 0.064 & 0.019 & 1.643 & 30.923 & 1136.941 \\
\hline BHI & 1.000 & 0.098 & 0.060 & 3.284 & 23.106 & 603.142 \\
\hline $\mathrm{BAC}$ & 0.477 & 0.054 & 0.015 & 1.681 & 20.789 & 578.406 \\
\hline BAX & 0.447 & 0.059 & 0.022 & 1.884 & 22.785 & 609.476 \\
\hline BDX & 0.481 & 0.036 & 0.021 & 1.109 & 19.322 & 541.972 \\
\hline BBBY & 1.078 & 0.029 & 0.051 & 1.579 & 7.060 & 92.898 \\
\hline BMY & 0.565 & 0.051 & 0.028 & 2.005 & 19.683 & 450.186 \\
\hline CPB & 0.470 & 0.083 & 0.012 & 1.940 & 38.241 & 1616.776 \\
\hline $\mathrm{COF}$ & 0.909 & 0.092 & 0.023 & 3.300 & 20.993 & 547.443 \\
\hline CAH & 0.465 & 0.044 & 0.016 & 1.705 & 18.142 & 393.755 \\
\hline CTL & 0.492 & 0.069 & 0.026 & 2.049 & 23.613 & 687.584 \\
\hline CTAS & 1.104 & 0.113 & 0.018 & 2.839 & 31.100 & 1212.341 \\
\hline C & 0.624 & 0.086 & 0.021 & 2.305 & 27.085 & 952.213 \\
\hline CLX & 0.481 & 0.074 & 0.026 & 2.203 & 27.445 & 840.327 \\
\hline CMS & 0.764 & 0.083 & 0.034 & 2.854 & 20.911 & 544.730 \\
\hline KO & 0.314 & 0.009 & 0.015 & 0.464 & 7.565 & 101.565 \\
\hline CL & 0.401 & 0.058 & 0.026 & 1.569 & 27.867 & 957.179 \\
\hline CMA & 0.345 & 0.025 & 0.011 & 1.011 & 16.800 & 350.599 \\
\hline $\mathrm{CSC}$ & 0.764 & 0.105 & 0.031 & 2.884 & 25.713 & 861.526 \\
\hline $\mathrm{CAG}$ & 0.530 & 0.072 & 0.014 & 2.129 & 25.460 & 758.407 \\
\hline COST & 0.773 & 0.135 & 0.042 & 3.358 & 33.314 & 1277.539 \\
\hline DOV & 0.441 & 0.050 & 0.031 & 1.334 & 27.171 & 949.990 \\
\hline DOW & 0.474 & 0.033 & 0.018 & 0.994 & 20.169 & 609.262 \\
\hline DTE & 0.296 & 0.068 & 0.017 & 1.552 & 41.597 & 1818.052 \\
\hline EMN & 0.432 & 0.089 & 0.027 & 2.141 & 35.535 & 1433.408 \\
\hline EIX & 1.103 & 0.251 & 0.022 & 6.892 & 26.622 & 885.136 \\
\hline ETR & 0.332 & 0.029 & 0.018 & 0.779 & 26.216 & 937.670 \\
\hline FDO & 0.911 & 0.093 & 0.043 & 2.519 & 26.543 & 911.464 \\
\hline FISV & 0.913 & 0.063 & 0.041 & 1.715 & 24.450 & 850.641 \\
\hline F & 0.635 & 0.024 & 0.050 & 1.053 & 10.141 & 170.597 \\
\hline GCI & 0.272 & 0.010 & 0.015 & 0.370 & 12.616 & 289.030 \\
\hline GPS & 1.007 & 0.049 & 0.032 & 2.730 & 10.649 & 142.751 \\
\hline $\mathrm{GE}$ & 0.445 & 0.037 & 0.013 & 1.233 & 19.948 & 515.188 \\
\hline GIS & 0.231 & 0.008 & 0.013 & 0.334 & 9.989 & 181.369 \\
\hline GPC & 0.427 & 0.089 & 0.018 & 2.039 & 40.541 & 1745.937 \\
\hline HNZ & 1.240 & 0.075 & 0.030 & 2.547 & 19.600 & 511.929 \\
\hline HPQ & 0.291 & 0.030 & 0.014 & 0.918 & 24.562 & 727.020 \\
\hline HD & 0.902 & 0.047 & 0.022 & 1.965 & 11.388 & 195.042 \\
\hline HON & 0.643 & 0.078 & 0.028 & 2.418 & 23.919 & 682.976 \\
\hline
\end{tabular}

\begin{tabular}{|c|c|c|c|c|c|c|}
\hline \multicolumn{7}{|c|}{ Forecasting sample: July 18,2006 to July 18,2008 (500 observations) } \\
\hline Stock & Mean (e-03) & Max. & Min.(e-03) & Std.dev.(e-03) & Skewness & Kurtosis \\
\hline ABT & 0.191 & 0.004 & 0.020 & 0.263 & 8.887 & 122.525 \\
\hline AFL & 0.268 & 0.004 & 0.015 & 0.406 & 4.815 & 35.550 \\
\hline APD & 0.261 & 0.003 & 0.013 & 0.292 & 4.633 & 38.977 \\
\hline AA & 0.559 & 0.007 & 0.051 & 0.682 & 4.078 & 25.551 \\
\hline ALL & 0.232 & 0.002 & 0.012 & 0.304 & 3.423 & 18.970 \\
\hline AXP & 0.498 & 0.015 & 0.010 & 0.919 & 8.966 & 127.909 \\
\hline AIG & 0.523 & 0.010 & 0.017 & 1.020 & 4.375 & 27.892 \\
\hline ADI & 0.432 & 0.014 & 0.046 & 0.816 & 11.614 & 168.440 \\
\hline APOL & 0.939 & 0.079 & 0.035 & 4.173 & 14.929 & 261.548 \\
\hline $\mathrm{T}$ & 0.278 & 0.004 & 0.023 & 0.399 & 6.116 & 50.180 \\
\hline AZO & 0.303 & 0.010 & 0.016 & 0.517 & 13.313 & 242.173 \\
\hline $\mathrm{AVY}$ & 0.243 & 0.005 & 0.019 & 0.446 & 7.402 & 70.878 \\
\hline BHI & 0.506 & 0.009 & 0.079 & 0.563 & 8.511 & 108.889 \\
\hline $\mathrm{BAC}$ & 0.495 & 0.016 & 0.012 & 1.223 & 7.682 & 79.279 \\
\hline BAX & 0.190 & 0.004 & 0.020 & 0.306 & 7.569 & 80.297 \\
\hline BDX & 0.150 & 0.003 & 0.017 & 0.210 & 8.356 & 104.636 \\
\hline BBBY & 0.464 & 0.010 & 0.029 & 0.627 & 7.907 & 106.865 \\
\hline BMY & 0.303 & 0.007 & 0.017 & 0.521 & 7.153 & 72.678 \\
\hline CPB & 0.152 & 0.002 & 0.011 & 0.206 & 4.746 & 30.567 \\
\hline $\mathrm{COF}$ & 0.923 & 0.013 & 0.028 & 1.481 & 3.995 & 25.175 \\
\hline CAH & 0.186 & 0.005 & 0.011 & 0.294 & 9.695 & 135.019 \\
\hline CTL & 0.279 & 0.021 & 0.022 & 1.008 & 17.453 & 350.144 \\
\hline CTAS & 0.293 & 0.006 & 0.034 & 0.395 & 8.196 & 99.256 \\
\hline C & 0.648 & 0.014 & 0.019 & 1.276 & 5.411 & 41.978 \\
\hline CLX & 0.153 & 0.007 & 0.012 & 0.416 & 13.180 & 203.335 \\
\hline CMS & 0.219 & 0.003 & 0.020 & 0.242 & 5.684 & 49.133 \\
\hline KO & 0.112 & 0.002 & 0.005 & 0.181 & 7.972 & 78.506 \\
\hline CL & 0.125 & 0.004 & 0.015 & 0.212 & 11.526 & 172.718 \\
\hline CMA & 0.632 & 0.023 & 0.017 & 1.400 & 9.231 & 131.210 \\
\hline $\mathrm{CSC}$ & 0.285 & 0.005 & 0.025 & 0.412 & 6.514 & 62.089 \\
\hline CAG & 0.179 & 0.005 & 0.016 & 0.313 & 9.470 & 120.784 \\
\hline COST & 0.338 & 0.010 & 0.030 & 0.564 & 11.158 & 166.572 \\
\hline DOV & 0.245 & 0.003 & 0.025 & 0.327 & 5.352 & 39.200 \\
\hline DOW & 0.347 & 0.007 & 0.028 & 0.616 & 6.593 & 55.276 \\
\hline DTE & 0.177 & 0.002 & 0.015 & 0.202 & 4.481 & 34.565 \\
\hline EMN & 0.292 & 0.005 & 0.024 & 0.429 & 6.244 & 57.701 \\
\hline EIX & 0.223 & 0.005 & 0.026 & 0.320 & 7.899 & 96.229 \\
\hline ETR & 0.203 & 0.003 & 0.017 & 0.281 & 5.482 & 44.736 \\
\hline FDO & 0.770 & 0.017 & 0.033 & 1.332 & 7.121 & 70.492 \\
\hline FISV & 0.276 & 0.006 & 0.031 & 0.366 & 7.898 & 96.806 \\
\hline F & 0.802 & 0.009 & 0.091 & 1.101 & 4.107 & 23.288 \\
\hline GCI & 0.305 & 0.012 & 0.018 & 0.658 & 12.646 & 218.866 \\
\hline GPS & 0.564 & 0.007 & 0.028 & 0.681 & 4.354 & 30.343 \\
\hline GE & 0.213 & 0.016 & 0.016 & 0.718 & 19.569 & 416.499 \\
\hline GIS & 0.108 & 0.002 & 0.011 & 0.137 & 6.537 & 58.387 \\
\hline GPC & 0.182 & 0.002 & 0.017 & 0.189 & 5.554 & 49.714 \\
\hline HNZ & 0.309 & 0.003 & 0.043 & 0.280 & 4.217 & 28.816 \\
\hline HPQ & 0.121 & 0.002 & 0.016 & 0.138 & 5.344 & 43.491 \\
\hline HD & 0.289 & 0.005 & 0.024 & 0.387 & 6.301 & 64.372 \\
\hline HON & 0.394 & 0.004 & 0.042 & 0.469 & 3.953 & 25.552 \\
\hline
\end{tabular}




\section{Appendix C. Figures}

Figure C.2: Standard deviation of the IMP Monte Carlo estimated scalar parameters $\theta, \omega, \alpha$ and $\beta$ against the cross-section dimension ranging from 10 to 50. Results from the two simulation studies for $T=1000,2000$ jointly reported respectively in Panel (a) and (b).

(a) $T=1000$
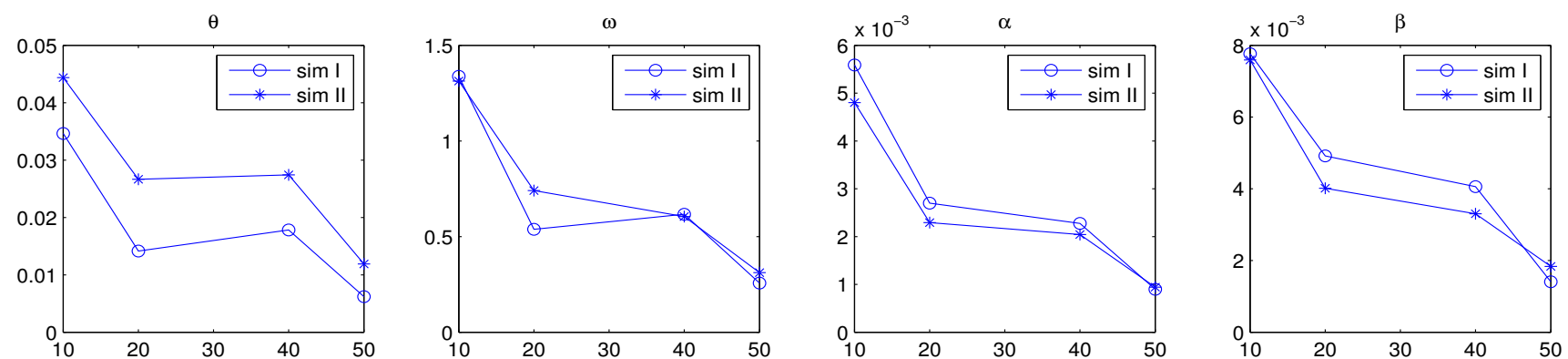

(b) $T=2000$
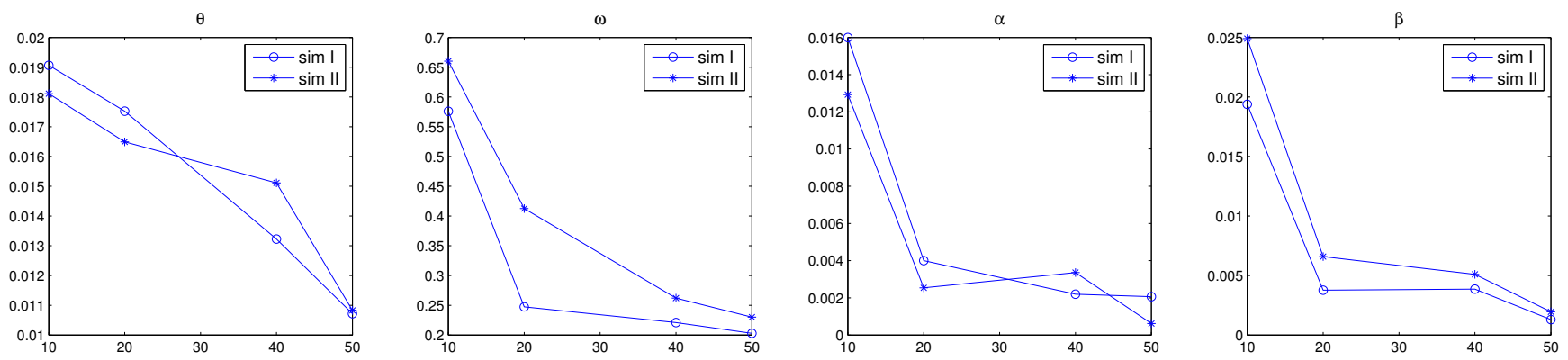
[1] Aielli, G. P. [2013], 'Dynamic conditional correlation: On properties and estimation', Journal of Business \& Economic Statistics 31, 282-299.

[2] Andersen, T., Bollerslev, T., Diebold, F. and Ebens, H. [2001], 'The distribution of realized stock return volatility', Journal of Financial Economics 61, 43-76.

[3] Barndorff-Nielsen, O. E., Hansen, P. R., Lunde, A. and Shephard, N. [2011], 'Multivariate realised kernels: consistent positive semi-definite estimators of the covariation of equity prices with noise and non-synchronous trading', Journal of Econometrics 162(2), 149-169.

[4] Barndorff-Nielsen, O. and Shephard, N. [2001], 'Normal modified stable processes', Theory of Probability and Mathematics Statistics 65, 1-19.

[5] Bauwens, L., Braione, M. and Storti, G. [2016], 'Forecasting comparison of long term component dynamic models for realized covariance matrices', Annals of Economics and Statistics, (Forthcoming) .

[6] Bauwens, L., Hafner, C. M. and Pierret, D. [2013], 'Multivariate volatility modeling of electricity futures', Journal of Applied Econometrics 28(5), 743-761.

[7] Bauwens, L., Storti, G. and Violante, F. [2012], Dynamic conditional correlation models for realized covariance matrices. CORE DP 2012/60.

[8] Bollerslev, T. [1990], 'Modeling the coherence in short-run nominal exchange rates: A multivariate generalized arch model', Review of Economics and Statistics 72, 498-505.

[9] Boswijk, H. P. [1995], 'Identifiability of cointegrated systems', Tinbergen Institute Working Paper (95/78).

[10] Boudt, K., Laurent, S., Lunde, A. and Quaedvlieg, R. [2014], 'Positive semidefinite integrated covariance estimation, factorizations and asynchronicity', Factorizations and Asynchronicity (October 8, 2014).

[11] Chiriac, R. and Voev, V. [2011], 'Modelling and forecasting multivariate realized volatility', Journal of Applied Econometrics 26, 922-947.

[12] Clements, M. P. and Smith, J. [1997], 'The performance of alternative forecasting methods for setar models', International Journal of Forecasting 13, 463-475.

[13] Cubadda, G. and Scambelloni, E. [2015], Index-augmented autoregressive models: representation, estimation and forecasting. Paper presented at the CFE 2015, London.

[14] Engle, R. [2002], 'Dynamic conditional correlation - a simple class of multivariate GARCH models', Journal of Business 86 Economic Statistics 20, 339-350. 
[15] Engle, R. F. [2009], 'High dimension dynamic correlations', The Methodology and Practice of Econometrics: A Festschrift in Honour of David F. Hendry: A Festschrift in Honour of David F. Hendry p. 122.

[16] Engle, R. F., Ghysels, E. and Sohn, B. [2013], 'Stock market volatility and macroeconomic fundamentals', Review of Economics and Statistics 95(3), 776-797.

[17] Engle, R. F., Shephard, N. and Sheppard, K. [2008], 'Fitting vast dimensional timevarying covariance models'.

[18] Engle, R. and Kelly, B. [2012], 'Dynamic equicorrelation', Journal of Business 83 Economic Statistics 30, 212-228.

[19] Fresoli, D. E. and Ruiz, E. [In Press], 'The uncertainty of conditional returns, volatilities and correlations in dec models', Computational Statistics $8 \mathcal{B}$ Data Analysis .

[20] Ghysels, E., Sinko, A. and Valkanov, R. [2007], 'Midas regressions: Further results and new directions', Econometric Reviews 26(1), 53-90.

[21] Golosnoy, V., Gribisch, B. and Liesenfeld, R. [2012], 'The conditional autoregressive wishart model for multivariate stock market volatility', Journal of Econometrics 167(1), 211-223.

[22] Gouriéroux, C., Jasiak, J. and Sufana, R. [2009], 'The wishart autoregressive process of multivariate stochastic volatility', Journal of Econometrics 150(2), 167-181.

[23] Hansen, P. R., Lunde, A. and Nason, J. M. [2011], 'The model confidence set', Econometrica 79(2), 453-497.

[24] Hautsch, N., Kyj, L. M. and Malec, P. [2015], 'Do high-frequency data improve highdimensional portfolio allocations?', Journal of Applied Econometrics 30(2), 263-290.

[25] Jin, X. and Maheu, J. M. [2013], 'Modeling realized covariances and returns', Journal of Financial Econometrics 11(2), 335-369.

[26] Laurent, S., Rombouts, J. V. and Violante, F. [2013], 'On loss functions and ranking forecasting performances of multivariate volatility models', Journal of Econometrics 173(1), 1-10.

[27] Morgan, J. [1994], 'Introduction to riskmetrics', New York: JP Morgan .

[28] Noureldin, D., Shephard, N. and Sheppard, K. [2012], 'Multivariate high-frequencybased volatility (heavy) models', Journal of Applied Econometrics 27(6), 907-933.

[29] Pascual, L., R. J. and Ruiz, E. [2006], 'Bootstrap prediction for returns and volatilities in garch models', Computational Statistics \&3 Data Analysis 50(9), 2293-2312. 
[30] Pedersen, R. S. and Rahbek, A. [2014], 'Multivariate variance targeting in the bekkgarch model', The Econometrics Journal 17(1), 24-55.

[31] Shephard, N. and Sheppard, K. K. [2010], 'Realising the future: forecasting with highfrequency-based volatility (heavy) models.', Journal of Applied Econometrics 25, 197231. 